\title{
Case Characteristics, Clinical Data, And Outcomes of Hospitalized COVID-19 Patients In Qom Province, Iran: A Prospective Cohort Study
}

Mohamad Amin Pourhoseingholi

Shahid Beheshti University of Medical Sciences

Hosein Yousefi

Qom University of Medical Science and Health Services

Hassan Fatemi Manesh

Qom University of Medical Science and Health Services

Nima Najafian Motahaver

Qom University of Medical Science and Health Services

Zahra Heydari

Qom University of Medical Science and Health Services

Mehdi Azizmohammad Looha

Shahid Beheshti University of Medical Sciences

Nazanin Taraghikhah

Shahid Beheshti University of Medical Sciences

Maryam Yazdi

Qom University of Medical Science and Health Services

Zahra Salami

Qom University of Medical Science and Health Services

Elmira Moallemi

Qom University of Medical Science and Health Services

Seyed Hasan Adeli ( $\nabla$ adeli@muq.ac.ir)

Qom University of Medical Science and Health Services

\section{Research Article}

Keywords: COVID-19, Pandemic, Iran, Risk factor, Cohort

Posted Date: May 26th, 2021

DOI: https://doi.org/10.21203/rs.3.rs-365321/v2

License: (c) (i) This work is licensed under a Creative Commons Attribution 4.0 International License. Read Full License 


\section{Abstract}

The outbreak of severe acute respiratory syndrome corona virus 2 (SARS-CoV-2) dates back to December 2019 in China. Iran has been one of the most virus inflicted countries. The aim of this study was to report demographics, signs and symptoms, laboratory findings, therapeutic approaches, and outcomes. This observational cohort study was performed from $20^{\text {th }}$ February 2020 to $20^{\text {th }}$ July 2020. Patients' information was recorded in their medical files. Multivariable analysis was performed to assess demographics, signs and symptoms, paraclinical data, treatments, outcomes of disease, and finding the risk factors of death subject to COVID-19. Of all 2468 participants, the mean age was $57.9 \pm 17.6$ years and $56.8 \%$ of patients were male. The most significant comorbidities were seen among those who have Hypertension and Diabetes Mellitus. $14.42 \%$ were admitted to ICU, and $17.2 \%$ died in hospital. The significant risk factors of death were ageing, male gender, HTN, CHF, CVA, CKD, increasing ESR, PT, WBC, liver function tests, and decreasing Oxygen saturation. Incontinent results in the case of COVID-19 outcomes and deathrelated risk factors attribute to marked differences in demographics and health care systems. The patients with hazardous risk factors must be detected urgently and monitored closely to save more lives.

\section{Introduction}

Severe acute respiratory syndrome corona virus 2 (SARS-CoV-2) was discovered as an infectious pathologic agent following a widespread outbreak in Wuhan city, the capital of Hubei province of China, in December 2019. [1].

At the outset, fever and respiratory were deemed the major symptoms of this storage virus (2). As time went by, the virus caused other serious clinical manifestations ranging from asymptomatic or mild constitutional symptoms to life-threatening complications leading to hospitalization and even death (3).

The rapid spread of SARS-CoV-2 to other countries including Japan, USA, Italy, Russia, Iran, South Korea revealed that COVID-19 is a highly contagious disease in that it caused a pandemic (4).

Iran has been one of the most prone countries to the virus of all [2]. It is approximately 1459370 identified COVID-19 patients by nasopharyngeal and oropharyngeal swabs polymerase chain reaction (PCR) test alongside with 58412 deaths (mortality rate: $4.00 \%$ ) have been recorded in Iran until February 06, 2021[3]. The first confirmed case of SARS-CoV-2 in Iran that was officially reported belonged to Qom, a holy city in the north-central Iran, on 19th February, 2020. At the onset of coronavirus disease-2019 (COVID-19) outburst, Qom proved to be not only the foremost city with regard to increasing number of patients and deaths among all cities in Middle East but also the source of sufficient and proper virus- related awareness and studies[4].

In an observational study from China, men figured the most hospitalized cases, ,with a median age of 56 years, a $26 \%$ intensive care unit (ICU) admission and $28 \%$ mortality rate [5]. Whereas, an Iranian article reported different figures in that the mean age of $50.75 \pm 19.33$ years, ICU admission and mortality rate of $8 \%$ and $10.8 \%$, respectively were recorded [6]. Definitely, various information attributes to the differences between countries in population demographic data, genetic, prevalence of comorbidities, and health care systems[7]. Reporting the clinical manifestations, risk factors, and outcomes of COVID-19 is essential to improve our knowledge and managerial skills related to the patients.

There was limited information to describe the characteristics and outcomes of Iranian hospitalized patients in relation to COVID-19-. This study aims to describe detailed demographics, comorbidities, signs and symptoms, para clinical tests, therapeutic protocols, severity of disease, death risk factors, outcomes of the hospitalized patients, 
and the follow up of post-discharge COVID-19 cases.The data gathered from academic health care centers in Qom, Iran, at the outset of COVID-19 pandemic crisis.

\section{Methods}

This prospective cohort study was conducted at four hospitals (Kamkar, Forghani, Beheshti, and Imam Reza) affiliated with the Qom University of Medical Sciences. Kamkar hospital was the first center in the Qom to admit the first case of COVID-19 in Iran.

We prospectively traced patients who admitted to all four hospitals from February 20 to July 20, 2020 the infectious cases diagnosed by nasopharyngeal or oropharyngeal swab PCR tests and spiral chest CT scan. All patients admitted according to world health organization (WHO), Centers for Disease Control and Prevention (CDC) and Iran's national guidelines. Furthermore, hypoxemic patients received different types of therapeutic agents and respiratory facilities included nasal cannula, simple facemask, facemask with reserve bag, noninvasive ventilation (NIV) and invasive ventilation based on the severity of hypoxemia during hospitalization. Serious patients admitted to ICUs while those who are required non-invasive oxygen therapy admitted to general wards.

The Qom University of medical sciences ethics committee (IR.MUQ.REC.1399.013) approved this cohort study. The procedures were in accordance with the ethical standards of the Qom University of medical sciences ethics committee and with the Helsinki Declaration of the World Medical Association. Informed consent was taken from all participants.

Patients were confirmed based on their PCR test, spiral chest CT scan findings, and clinical presentations. Second confirming PCR tests were performed on either highly suspicious clinical presentations patients or those with falsenegative PCR tests coming from insufficient sample collection. There were no cases transfer among hospitals. For readmission cases, the first admission data were recorded. The data were collected only from COVID-19 cases whose full-length hospital stay (died or discharged) was available during the study.

Two criteria including 1.02sat $\geq 93$ percentage without Oxygen support, and 2. Normal body temperature for 2-3 days without any anti-pyretic drugs were considered for discharging patients.

Patients' demographics, comorbidities, exposure history, signs and symptoms, vital signs, laboratory data, CT scan findings, therapeutic approaches, duration of hospitalization, and outcomes were documented in their paper medical records. Afterwards, these data were collected as the standard data collection form and then rechecked by both a physician and a statistician. In case of any disagreements, they were reassessed by a third physician. A 30day telephone investigation of post-discharged patients was taken into account after patients' discharge. Patients were asked about the existence of symptoms, relapse of symptoms, and the requirement of readmission and the occurrence of death by the telephone investigation.

Symptoms, vital signs, radiological findings, laboratory data and type of respiratory facilities were defined within the first day of admission.

All data regarding demographics, exposure history, co-morbidities, signs and symptoms, Chest CT scan and laboratory findings were collected within 24 hours of admission. Additionally, the data with respect to therapeutic interventions including supplemental oxygen (facemask with or without reserve bag, noninvasive or invasive 
ventilation), administration of anti-viral agents, anti-bacterial agents, immunomodulatory agents, hemodialysis and therapeutic plasma exchange were recorded. Finally, data collected from post-discharge follow up.

All diagnostic real-time reverse-transcriptase-polymerase chain-reaction (RT-PCR) tests of nasopharyngeal and oropharyngeal specimens were performed in all of these four hospitals.

The main outcome of this study was to define the rate of death and survive in hospitalized patients. The secondary outcomes were frequency of demographics, comorbidities, signs and symptoms, respiratory facilities (invasive or non-invasive), drugs, laboratory data, and their association with the severity of disease and mortality.

Descriptive statistics were regarded as mean \pm SD or median (interquartile range) for continuous data and frequency (percentage) for categorical data. The chi-square independent was used to determine whether there is a significant relationship between categorical variables. The independent t-test and Mann-Whitney U test were applied to compare differences of continuous variables between groups. Graphs including Euler diagram and Heat map were utilized to represent the relationship between groups and the different frequencies respectively.

The death outcome as the event of interest for survival analysis was considered the time interval between hospitalization and event (death or discharged) was deemed as the survival time. Discharged Patients were regarded as the censored cases. The single and multiple Cox regression were used to evaluate the single and adjusted effect of risk factors on the survival time. Adjusted model was chosen with the help of stepwise selection method with forward approach. Kaplan-Meier survival curve was applied to illustrate cumulative probability of occurring event of interest. All analyzes were performed by R 3.6.2. P-values less than 0.05 were considered as the statistically significant.

\section{Results}

2468 patients with clinical presentations, chest CT scan findings that were confirmed by positive RT-PCR tests, based on Iran's national guidelines, were admitted to the hospitals between February 20 and July 20, 2020.

Table 1 summarized the baseline characteristics and their association with disease outcome (death or survive) and severity. The mean age of patients was $57.9 \pm 17.6$ years while the mean age of the dead was significantly higher than survivors $(66.8 \pm 15.0$ vs $56.0 \pm 17.6$, p-value $<0.001)$. Although all age groups probable infected with SARSCoV-2, the highest rate of infection belonged to 60-69 years age group. The mean BMI of patients was $25.4 \pm 3.2$ $\mathrm{kg} / \mathrm{m}^{2}$ of which $55.2 \%$ were overweight and obese, 1402 (56.8\%) were men, 2291(92.9\%) were Iranian and 2354 (95.4\%) were non-smokers while $2167(87.8 \%)$ of cases were reported none or unknown history of exposure. $5.4 \%$ of cases had misused Antibiotics before admission.

Table 1.Baseline characteristics of patients with COVID-19 


\begin{tabular}{|c|c|c|c|c|c|c|c|}
\hline \multirow[t]{2}{*}{ Variables } & \multirow{2}{*}{$\begin{array}{l}\text { Total } \\
(n=2468)\end{array}$} & \multicolumn{2}{|c|}{ Disease Outcome } & \multirow[t]{2}{*}{$P$-value } & \multicolumn{2}{|l|}{ Ward } & \multirow[t]{2}{*}{$P$-value } \\
\hline & & $\begin{array}{l}\text { Deceased } \\
(n=424)\end{array}$ & $\begin{array}{l}\text { Survivor } \\
(n=2044)\end{array}$ & & $\begin{array}{l}\text { General } \\
(\mathrm{n}=2112)\end{array}$ & $\begin{array}{l}\text { ICU } \\
(n=356)\end{array}$ & \\
\hline \multicolumn{8}{|l|}{ Age } \\
\hline Mean \pm SD & $57.9(17.6)$ & $\begin{array}{l}66.8 \\
(15.0)\end{array}$ & $56.0(17.6)$ & $<0.001^{\mathrm{a}}$ & $56.8(19.6)$ & $\begin{array}{l}63.9 \\
(16.5)\end{array}$ & $<0.001^{\mathrm{a}}$ \\
\hline Median (Q1 - Q3) & $\begin{array}{l}59.0(45.0 \\
-71.0)\end{array}$ & $\begin{array}{l}68.0 \\
(58.0- \\
78.0)\end{array}$ & $\begin{array}{l}57.0(42.0- \\
69.0)\end{array}$ & $<0.001^{b}$ & $\begin{array}{l}58.0(44.0 \\
-70.0)\end{array}$ & $\begin{array}{l}65.0 \\
(53.0- \\
77.0)\end{array}$ & $<0.001^{b}$ \\
\hline $0-9$ & $0(0)$ & $0(0)$ & $0(0)$ & $<0.001^{\mathrm{c}}$ & $0(0)$ & $0(0)$ & $<0.001^{\circ}$ \\
\hline 10-19 & $34(1.4)$ & $0(0)$ & $34(1.7)$ & & $33(1.6)$ & $1(0.3)$ & \\
\hline $20-29$ & $123(5)$ & $7(1.7)$ & $116(5.7)$ & & $112(5.3)$ & $11(3.1)$ & \\
\hline $30-39$ & $271(11)$ & $10(2.4)$ & $261(12.8)$ & & $251(11.9)$ & $20(5.6)$ & \\
\hline $40-49$ & $365(14.8)$ & $47(11.1)$ & $318(15.6)$ & & $327(15.5)$ & $38(10.7)$ & \\
\hline $50-59$ & 483 (19.6) & $60(14.2)$ & $423(20.7)$ & & $422(20)$ & $61(17.1)$ & \\
\hline $60-69$ & $513(20.8)$ & $\begin{array}{l}101 \\
(23.8)\end{array}$ & $412(20.2)$ & & $433(20.5)$ & $80(22.5)$ & \\
\hline 70-79 & 386 (15.6) & $\begin{array}{l}111 \\
(26.2)\end{array}$ & 275 (13.5) & & 307 (14.5) & 79 (22.2) & \\
\hline $80-89$ & $246(10)$ & $69(16.3)$ & $177(8.7)$ & & $193(9.1)$ & $53(14.9)$ & \\
\hline 90-99 & $47(1.9)$ & $19(4.5)$ & $28(1.4)$ & & $34(1.6)$ & $13(3.7)$ & \\
\hline Gender No (\%) & & & & $0.039^{c}$ & & & $0.929^{c}$ \\
\hline Female & $\begin{array}{l}1066 \\
(43.2)\end{array}$ & $\begin{array}{l}164 \\
(38.7)\end{array}$ & $902(44.1)$ & & $913(43.2)$ & $\begin{array}{l}153 \\
(43.0)\end{array}$ & \\
\hline Male & $\begin{array}{l}1402 \\
(56.8)\end{array}$ & $\begin{array}{l}260 \\
(61.3)\end{array}$ & $\begin{array}{l}1142 \\
(55.9)\end{array}$ & & $\begin{array}{l}11199 \\
(56.8)\end{array}$ & $\begin{array}{l}203 \\
(57.0)\end{array}$ & \\
\hline Pregnancy, N (\%)* & & & & $0.211^{c}$ & & & $0.760^{c}$ \\
\hline Yes & $32(3.0)$ & $\begin{array}{l}2 / 164 \\
(1.2)\end{array}$ & $\begin{array}{l}30 / 902 \\
(3.3)\end{array}$ & & $\begin{array}{l}28 / 913 \\
(3.1)\end{array}$ & $\begin{array}{l}6 / 153 \\
(3.9 \%)\end{array}$ & \\
\hline \multicolumn{8}{|l|}{ BMI } \\
\hline Mean \pm SD & $25.4(3.2)$ & $25.6(3.1)$ & $25.3(3.2)$ & $0.055^{a}$ & $25.3(3.2)$ & $\begin{array}{l}25.5 \\
(3.1)\end{array}$ & $0.421^{c}$ \\
\hline $\begin{array}{l}\text { Underweight (= } \\
<18.5)\end{array}$ & $10(0.4)$ & $2(0.5)$ & $8(0.4)$ & $0.043^{c}$ & $9(0.4)$ & $1(0.3)$ & $0.496^{c}$ \\
\hline $\begin{array}{l}\text { Normal weight }(= \\
18.5-24.9)\end{array}$ & $\begin{array}{l}1097 \\
(44.4)\end{array}$ & $\begin{array}{l}163 \\
(38.4)\end{array}$ & $934(45.7)$ & & $947(44.8)$ & $\begin{array}{l}150 \\
(42.1)\end{array}$ & \\
\hline $\begin{array}{l}\text { Overweight (= 25- } \\
29.9)\end{array}$ & $\begin{array}{l}1211 \\
(49.1)\end{array}$ & $\begin{array}{l}234 \\
(55.2)\end{array}$ & $977(47.8)$ & & 1024 (48.5) & $\begin{array}{l}187 \\
(52.5)\end{array}$ & \\
\hline
\end{tabular}




\begin{tabular}{|c|c|c|c|c|c|c|c|}
\hline $\begin{array}{l}\text { Obesity (30 or } \\
\text { greater) }\end{array}$ & $150(6.1)$ & $25(5.9)$ & $125(6.1)$ & & $132(6.3)$ & $18(5.1)$ & \\
\hline Location, No (\%) & & & & $0.051^{c}$ & & & $0.078^{c}$ \\
\hline Village & $103(4.2)$ & $25(5.9)$ & $78(3.8)$ & & $82(3.9)$ & $21(5.9)$ & \\
\hline City & $\begin{array}{l}2365 \\
(95.8)\end{array}$ & $\begin{array}{l}399 \\
(94.1)\end{array}$ & $\begin{array}{l}1966 \\
(96.2)\end{array}$ & & $2030(96.1)$ & $\begin{array}{l}335 \\
(94.1)\end{array}$ & \\
\hline Nation, NO (\%) & & & & $0.545^{c}$ & & & $0.291^{c}$ \\
\hline Other & $175(7.1)$ & $33(7.8)$ & $142(7.0)$ & & $145(6.9)$ & $30(8.4)$ & \\
\hline Iran & $\begin{array}{l}2291 \\
(92.9)\end{array}$ & $\begin{array}{l}391 \\
(92.2)\end{array}$ & $\begin{array}{l}1900 \\
(93.0)\end{array}$ & & 1965 (93.1) & $\begin{array}{l}326 \\
(91.6)\end{array}$ & \\
\hline $\begin{array}{l}\text { Exposure History, } \\
\text { No (\%) }\end{array}$ & & & & $0.936^{c}$ & & & $0.800^{c}$ \\
\hline No or Unknown & $\begin{array}{l}2167 \\
(87.8)\end{array}$ & $\begin{array}{l}368 \\
(86.8)\end{array}$ & 1799 (88) & & $1860(88.1)$ & $\begin{array}{l}307 \\
(86.2)\end{array}$ & \\
\hline Household contact & $170(6.9)$ & $36(8.5)$ & $134(6.6)$ & & $143(6.8)$ & $27(7.6)$ & \\
\hline $\begin{array}{l}\text { Transmission out } \\
\text { of Home }\end{array}$ & $125(5.1)$ & $20(4.7)$ & $105(5.1)$ & & $104(4.9)$ & $21(5.9)$ & \\
\hline Travelling to China & $6(0.2)$ & $0(0)$ & $6(0.3)$ & & $5(0.2)$ & $1(0.3)$ & \\
\hline \multicolumn{8}{|l|}{$\begin{array}{l}\text { Comorbidity, No of } \\
\text { yes (\%) }\end{array}$} \\
\hline $\begin{array}{l}\text { Hypertension } \\
\text { (HTN) }\end{array}$ & $773(31.3)$ & $\begin{array}{l}167 \\
(39.4)\end{array}$ & $606(29.6)$ & $<0.001^{\mathrm{c}}$ & $633(30)$ & $\begin{array}{l}140 \\
(39.3)\end{array}$ & $<0.001^{c}$ \\
\hline $\begin{array}{l}\text { Ischemic heart } \\
\text { disease (IHD) }\end{array}$ & $259(10.5)$ & $49(11.6)$ & $210(10.3)$ & $0.443^{c}$ & 218 (10.3) & $41(11.5)$ & $0.496^{c}$ \\
\hline $\begin{array}{l}\text { Coronary artery } \\
\text { bypass grafting } \\
\text { (CABG) }\end{array}$ & $71(2.9)$ & $18(4.2)$ & $53(2.6)$ & $0.064^{c}$ & $59(2.8)$ & $12(3.4)$ & $0.547^{c}$ \\
\hline $\begin{array}{l}\text { Cognitive hear } \\
\text { failure (CHF) }\end{array}$ & $37(1.5)$ & $18(4.2)$ & $19(0.09)$ & $<0.001^{c}$ & $25(1.2)$ & $12(3.4)$ & $0.002^{c}$ \\
\hline Asthma & $100(4.1)$ & $13(3.1)$ & $87(4.3)$ & $0.258^{c}$ & $84(4)$ & $16(4.5)$ & $0.647^{c}$ \\
\hline $\begin{array}{l}\text { Chronic } \\
\text { obstructive } \\
\text { pulmonary disease } \\
\text { (COPD) }\end{array}$ & $100(4.1)$ & $30(7.1)$ & $70(3.4)$ & $0.001^{c}$ & $83(3.9)$ & $17(4.8)$ & $0.454^{c}$ \\
\hline $\begin{array}{l}\text { Diabetes mellitus } \\
\text { (DM) }\end{array}$ & 679 (27.5) & $\begin{array}{l}145 \\
(34.2)\end{array}$ & $534(26.1)$ & $0.001^{c}$ & $552(26.1)$ & $\begin{array}{l}127 \\
(35.7)\end{array}$ & $<0.001^{\mathrm{C}}$ \\
\hline Pneumonia & $62(2.5)$ & $17(4.0)$ & $45(2.2)$ & $0.030^{c}$ & $49(2.3)$ & $13(3.7)$ & $0.137^{c}$ \\
\hline $\begin{array}{l}\text { Cerebrovascular } \\
\text { accident (CVA) }\end{array}$ & $61(2.5)$ & $19(4.5)$ & $42(2.1)$ & $0.003^{c}$ & $47(2.2)$ & $14(3.9)$ & $0.055^{c}$ \\
\hline $\begin{array}{l}\text { Gastrointestinal } \\
(\mathrm{Gl})\end{array}$ & $68(2.8)$ & $13(3.1)$ & $55(2.7)$ & $0.668^{c}$ & $58(2.7)$ & $10(2.8)$ & $0.947^{c}$ \\
\hline
\end{tabular}




\begin{tabular}{|c|c|c|c|c|c|c|c|}
\hline $\begin{array}{l}\text { Chronic kidney } \\
\text { disease (CKD) }\end{array}$ & $89(3.6)$ & $31(7.3)$ & $58(2.8)$ & $<0.001^{c}$ & $70(3.3)$ & $19(5.3)$ & $0.058^{c}$ \\
\hline $\begin{array}{l}\text { Rheumatoid } \\
\text { arthritis (RA) }\end{array}$ & $76(3.1)$ & $16(3.8)$ & $60(2.9)$ & $0.363^{c}$ & $66(3.1)$ & $10(2.8)$ & $0.750^{c}$ \\
\hline Cancer & $37(1.5)$ & $14(3.3)$ & $23(1.1)$ & $0.001^{\mathrm{c}}$ & $28(1.3)$ & $9(2.5)$ & $0.084^{c}$ \\
\hline $\begin{array}{l}\text { Hyperlipidemia } \\
\text { (HLP) }\end{array}$ & 322 (13.0) & 65 (15.3) & 257 (12.6) & $0.125^{c}$ & $280(13.3)$ & $42(11.8)$ & $0.449^{c}$ \\
\hline $\begin{array}{l}\text { Hepatitis C virus } \\
(\mathrm{HCV})\end{array}$ & $17(0.7)$ & $4(0.9)$ & $13(0.6)$ & $0.515^{c}$ & $15(0.7)$ & $2(0.6)$ & $0.754^{c}$ \\
\hline Thyroid disease & $60(2.4 \%)$ & $9(2.1)$ & $51(2.5)$ & $0.650^{c}$ & $53(2.5)$ & $7(2)$ & $0.538^{c}$ \\
\hline $\begin{array}{l}\text { Other Immune } \\
\text { deficiencies }\end{array}$ & $38(1.5)$ & $5(1.2)$ & $33(1.6)$ & $0.508^{c}$ & $33(1.6)$ & $5(1.4)$ & $0.823^{c}$ \\
\hline Hysteria & $32(1.3)$ & $8(1.9)$ & $24(1.2)$ & $0.328^{c}$ & $24(1.1)$ & $8(2.2)$ & $0.087^{c}$ \\
\hline Tuberculosis & $31(1.3)$ & $7(1.7)$ & $24(1.2)$ & $0.422^{c}$ & $26(1.2)$ & $5(1.4)$ & $0.786^{c}$ \\
\hline Anemia & $29(1.2)$ & $5(1.2)$ & $24(1.2)$ & $0.993^{c}$ & $24(1.1)$ & $5(1.4)$ & $0.664^{c}$ \\
\hline Fatty liver & 105 (4.3) & $78(4.2)$ & $87(4.3)$ & $0.992^{c}$ & $86(4.1)$ & $19(5.3)$ & $0.274^{c}$ \\
\hline $\begin{array}{l}\text { Other Neurological } \\
\text { disorders }\end{array}$ & $34(1.4)$ & $6(1.4)$ & $28(1.4)$ & $0.942^{c}$ & $30(1.4)$ & $4(1.1)$ & $0.657^{c}$ \\
\hline Parkinson & $11(0.4)$ & $2(0.5)$ & $9(0.4)$ & $0.930^{c}$ & $9(0.4)$ & $2(0.6)$ & $0.722^{c}$ \\
\hline Alzheimer & $23(0.9)$ & $6(1.4)$ & $17(0.8)$ & $0.255^{c}$ & $19(0.9)$ & $4(1.1)$ & $0.684^{c}$ \\
\hline Smoke, N (\%) & & & & $0.719^{c}$ & & & $0.347^{c}$ \\
\hline Yes & $114(4.6)$ & $21(5.0)$ & $93(4.5)$ & & $101(4.8)$ & $13(3.7)$ & \\
\hline $\begin{array}{l}\text { Opium addiction, } \\
\mathrm{N}(\%)\end{array}$ & & & & $0.309^{c}$ & & & $0.051^{c}$ \\
\hline Yes & $28(1.1)$ & $7(1.7)$ & $21(1.0)$ & & $20(0.9)$ & $8(2.2)$ & \\
\hline \multicolumn{8}{|l|}{$\begin{array}{l}\text { Improper use of } \\
\text { drugs, } \mathbf{N}(\%)\end{array}$} \\
\hline $\begin{array}{l}\text { Proton-pump } \\
\text { inhibitors (PPIs) }\end{array}$ & $323(13.1)$ & $60(14.2)$ & $263(12.9)$ & $0.476^{c}$ & $271(12.8)$ & $52(14.6)$ & $0.354^{c}$ \\
\hline $\begin{array}{l}\text { Nonsteroidal anti- } \\
\text { inflammatory drug } \\
\text { (NSAID) }\end{array}$ & $147(6.0)$ & $20(4.7)$ & $127(6.2)$ & $0.236^{c}$ & $133(6.3)$ & $14(3.9)$ & $0.081^{c}$ \\
\hline $\begin{array}{l}\text { Antibiotics (oral } \\
\text { agents) }\end{array}$ & $133(5.4)$ & $34(8.0)$ & $99(4.8)$ & $0.008^{c}$ & $79(3.7)$ & $54(15.2)$ & $<0.001^{c}$ \\
\hline
\end{tabular}




\begin{tabular}{|c|c|c|c|c|c|c|c|}
\hline Mean (SD) & $7.4(3.7)$ & $7.4(3.7)$ & $7.4(3.7)$ & $0.986^{\mathrm{a}}$ & $7.4(3.7)$ & $7.2(3.6)$ & $0.247^{a}$ \\
\hline Median (IQR) & $\begin{array}{l}7.0(5.0- \\
10.0)\end{array}$ & $\begin{array}{l}7.0(5.0- \\
10.0)\end{array}$ & $\begin{array}{l}7.0(5.0- \\
10.0)\end{array}$ & $0.930^{\mathrm{b}}$ & $\begin{array}{l}7.0(5.0- \\
10.0)\end{array}$ & $\begin{array}{l}7.0(5.0 \\
-9.0)\end{array}$ & $0.263^{b}$ \\
\hline \multicolumn{8}{|l|}{$\begin{array}{l}\text { Sign and } \\
\text { symptoms, No. of } \\
\text { Yes (\%) }\end{array}$} \\
\hline $\begin{array}{l}\text { Fever ( } \\
\text { temperature } \geq \\
37.8^{\circ} \mathrm{C}(\end{array}$ & $\begin{array}{l}1439 \\
(58.3)\end{array}$ & $\begin{array}{l}253 \\
(59.7)\end{array}$ & $1186(58)$ & $0.532^{c}$ & 1244 (58.9) & $\begin{array}{l}195 \\
(54.8)\end{array}$ & $0.144^{c}$ \\
\hline Cough & $\begin{array}{l}1868 \\
(75.7)\end{array}$ & $\begin{array}{l}305 \\
(71.9)\end{array}$ & $\begin{array}{l}1563 \\
(76.5)\end{array}$ & $0.048^{c}$ & 1627 (77) & $\begin{array}{l}241 \\
(67.7)\end{array}$ & $<0.001^{c}$ \\
\hline Throat clearing & $283(11.5)$ & $45(10.6)$ & 238 (11.6) & $0.544^{c}$ & $250(11.8)$ & $33(9.3)$ & $0.160^{c}$ \\
\hline Dyspnea & $\begin{array}{l}1944 \\
(78.8)\end{array}$ & $\begin{array}{l}331 \\
(78.1)\end{array}$ & $\begin{array}{l}1613 \\
(78.9)\end{array}$ & $0.698^{c}$ & 1646 (77.9) & $\begin{array}{l}298 \\
(83.7)\end{array}$ & $0.014^{c}$ \\
\hline $\begin{array}{l}\text { Myalgia or } \\
\text { arthralgia }\end{array}$ & $580(23.5)$ & 58 (13.7) & $522(25.5)$ & $\begin{array}{l}<0.001 \\
c\end{array}$ & $517(24.5)$ & 63 (17.7) & $0.005^{c}$ \\
\hline Fatigue & $584(23.7)$ & $\begin{array}{l}106 \\
(25.0)\end{array}$ & 478 (23.4) & $0.477^{c}$ & $496(23.5)$ & 88 (24.7) & $0.612^{c}$ \\
\hline Headache & $172(7.0)$ & $17(4.0)$ & $155(7.6)$ & $0.009^{c}$ & 155 (7.3) & $17(4.8)$ & $0.079^{c}$ \\
\hline Nausea & 505 (20.5) & 79 (18.6) & $426(20.8)$ & $0.305^{c}$ & 441 (20.9) & 64 (18) & $0.209^{c}$ \\
\hline Diarrhea & $182(7.4)$ & $21(5.0)$ & $161(7.9)$ & $0.036^{c}$ & $165(7.8)$ & $17(4.8)$ & $0.043^{c}$ \\
\hline Abdominal pain & $133(5.4)$ & $23(5.4)$ & $110(5.4)$ & $0.972^{c}$ & $115(5.4)$ & $18(5.1)$ & $0.764^{c}$ \\
\hline Dizziness & $79(3.2)$ & $9(2.1)$ & $70(3.4)$ & $0.166^{c}$ & $72(3.4)$ & $7(2)$ & $0.153^{c}$ \\
\hline Parosmia & $246(10)$ & $44(10.4)$ & 202 (9.9) & $0.757^{c}$ & 204 (9.7) & $42(11.8)$ & $0.213^{c}$ \\
\hline Anorexia & $416(16.9)$ & $61(14.4)$ & 355 (17.4) & $0.136^{c}$ & $362(17.1)$ & $54(15.2)$ & $0.358^{c}$ \\
\hline $\begin{array}{l}\text { loss of } \\
\text { consciousness }\end{array}$ & $61(2.5)$ & $23(5.4)$ & $38(1.9)$ & $<0.001^{\mathrm{c}}$ & $41(1.9)$ & $20(5.6)$ & $<0.001^{\mathrm{c}}$ \\
\hline Sweating & $33(1.3)$ & $2(0.5)$ & $31(1.5)$ & $0.088^{c}$ & $29(1.4)$ & $4(1.1)$ & $0.705^{c}$ \\
\hline Hemoptysis & $45(1.8)$ & $10(2.4)$ & $35(1.7)$ & $0.365^{c}$ & $39(1.8)$ & $6(1.7)$ & $0.833^{c}$ \\
\hline Hallucination & $51(2.1)$ & $7(1.7)$ & $44(2.2)$ & $0.509^{c}$ & $42(2)$ & $9(2.5)$ & $0.508^{c}$ \\
\hline \multicolumn{8}{|l|}{$\begin{array}{l}\text { Chest CT images, } \\
\text { No./total No. (\%) }\end{array}$} \\
\hline Normal & $\begin{array}{l}144 / 2468 \\
(5.8)\end{array}$ & $\begin{array}{l}23 / 424 \\
(5.4)\end{array}$ & $\begin{array}{l}121 / 2044 \\
(5.9)\end{array}$ & $0.692^{c}$ & $\begin{array}{l}123 / 2112 \\
(5.8)\end{array}$ & $\begin{array}{l}21 / 356 \\
(5.9)\end{array}$ & $0955^{c}$ \\
\hline Abnormal & $\begin{array}{l}2324 / 2468 \\
(94.2)\end{array}$ & $\begin{array}{l}401 / 424 \\
(94.6)\end{array}$ & $\begin{array}{l}1923 / 2044 \\
(94.12)\end{array}$ & & $\begin{array}{l}\text { 1989/2112 } \\
(94.2)\end{array}$ & $\begin{array}{l}335 / 356 \\
(94.1)\end{array}$ & \\
\hline Bilateral & $1453 / 2324$ & $256 / 401$ & $\begin{array}{c}\text { 1194/1923 } \\
\text { Page 8/25 }\end{array}$ & & 1238/1989 & $215 / 335$ & \\
\hline
\end{tabular}




\begin{tabular}{|c|c|c|c|c|c|c|c|}
\hline lung & $(94.2)$ & $(64.6)$ & $(62.1)$ & $0.347^{c}$ & $(62.2)$ & $(64.2)$ & $0.498^{c}$ \\
\hline Unilateral lung & $\begin{array}{l}871 / 2324 \\
(35.3)\end{array}$ & $\begin{array}{l}142 / 401 \\
(35.4)\end{array}$ & $\begin{array}{l}729 / 1923 \\
(37.9)\end{array}$ & $0.347^{c}$ & $\begin{array}{l}751 / 1989 \\
(37.8)\end{array}$ & $\begin{array}{l}120 / 335 \\
(35.8)\end{array}$ & $0.498^{c}$ \\
\hline $\begin{array}{c}\text { Ground } \\
\text { lass opacity }(G G O)\end{array}$ & $\begin{array}{l}2028 / 2324 \\
(94.2)\end{array}$ & $\begin{array}{l}365 / 401 \\
(91.0)\end{array}$ & $\begin{array}{l}1663 / 1923 \\
(86.5)\end{array}$ & $0.013^{c}$ & $\begin{array}{l}1725 / 1989 \\
(86.7)\end{array}$ & $\begin{array}{l}303 / 335 \\
(90.4)\end{array}$ & $0.059^{c}$ \\
\hline effusion Pleural & $\begin{array}{l}375 / 2324 \\
(15.2)\end{array}$ & $\begin{array}{l}137 / 401 \\
(34.2)\end{array}$ & $\begin{array}{l}238 / 1923 \\
(12.4)\end{array}$ & $<0.001^{\mathrm{c}}$ & $\begin{array}{l}264 / 1989 \\
(13.3)\end{array}$ & $\begin{array}{l}111 / 335 \\
(33.1)\end{array}$ & $<0.001^{c}$ \\
\hline effusion & $\begin{array}{l}315 / 2324 \\
(12.8)\end{array}$ & $\begin{array}{l}129 / 401 \\
(32.2)\end{array}$ & $\begin{array}{l}186 / 1923 \\
(9.7)\end{array}$ & $<0.001^{c}$ & $\begin{array}{l}209 / 1989 \\
(10.5)\end{array}$ & $\begin{array}{l}106 / 335 \\
(31.6)\end{array}$ & $<0.001^{c}$ \\
\hline Consolidation & $\begin{array}{l}729 / 2324 \\
(29.5)\end{array}$ & $\begin{array}{l}210 / 401 \\
(54.2)\end{array}$ & $\begin{array}{l}519 / 1923 \\
(27.0)\end{array}$ & $<0.001^{c}$ & $\begin{array}{l}506 / 1989 \\
(25.4)\end{array}$ & $\begin{array}{l}223 / 335 \\
(66.6)\end{array}$ & $<0.001^{c}$ \\
\hline paving Crazy & $\begin{array}{l}604 / 2324 \\
(26.0)\end{array}$ & $\begin{array}{l}158 / 401 \\
(39.4)\end{array}$ & $\begin{array}{l}446 / 1923 \\
(23.2)\end{array}$ & $<0.001^{c}$ & $\begin{array}{l}456 / 1989 \\
(22.9)\end{array}$ & $\begin{array}{l}148 / 335 \\
(44.2)\end{array}$ & $<0.001^{c}$ \\
\hline $\begin{array}{l}\text { Pericardial } \\
\text { effusion }\end{array}$ & $\begin{array}{l}112 / 2324 \\
(4.8)\end{array}$ & $\begin{array}{l}32 / 401 \\
(8.0)\end{array}$ & $\begin{array}{l}80 / 1923 \\
(4.2)\end{array}$ & $0.001^{c}$ & $\begin{array}{l}56 / 1989 \\
(2.8)\end{array}$ & $\begin{array}{l}56 / 335 \\
(16.7)\end{array}$ & $<0.001^{c}$ \\
\hline \multicolumn{8}{|l|}{ Vital sign } \\
\hline $\begin{array}{l}\text { Oxygen saturation } \\
\text { (SpO2) (normal } \\
\text { range } 90-92 \%)\end{array}$ & $\begin{array}{l}92(88- \\
95)\end{array}$ & $\begin{array}{l}88(80- \\
93)\end{array}$ & $\begin{array}{l}92(90- \\
95)\end{array}$ & $<0.001^{b}$ & $\begin{array}{l}92.0(89.3 \\
-95.0)\end{array}$ & $\begin{array}{l}88(80- \\
93)\end{array}$ & $<0.001^{b}$ \\
\hline $\begin{array}{l}\text { Systolic } \\
\text { pressure(normal } \\
\text { range } \leq 120 \mathrm{mmHg} \text { ) }\end{array}$ & $\begin{array}{l}130(110- \\
146)\end{array}$ & $\begin{array}{l}135(120 \\
-150)\end{array}$ & $\begin{array}{l}125(110- \\
145)\end{array}$ & $<0.001^{b}$ & $\begin{array}{l}126(110- \\
145)\end{array}$ & $\begin{array}{l}130(112 \\
-150)\end{array}$ & $0.010^{b}$ \\
\hline $\begin{array}{l}\text { Diastolic } \\
\text { pressure(normal } \\
\text { range } \leq 80 \mathrm{mmHg})\end{array}$ & $\begin{array}{l}80(70- \\
90)\end{array}$ & $\begin{array}{l}80(73- \\
90)\end{array}$ & $\begin{array}{l}80(70- \\
90)\end{array}$ & $<0.001^{b}$ & $80(70$ - 90) & $\begin{array}{l}80(70- \\
90)\end{array}$ & $0.006^{b}$ \\
\hline $\begin{array}{l}\text { Pulse rate (PR) } \\
\text { (normal range 60- } \\
100 \text { BPM) }\end{array}$ & $\begin{array}{l}87(80- \\
98)\end{array}$ & $\begin{array}{l}88(80- \\
100)\end{array}$ & $\begin{array}{l}86(80- \\
98)\end{array}$ & $0.043^{b}$ & $86(80$ - 98) & $\begin{array}{l}88(80- \\
100)\end{array}$ & $0.010^{b}$ \\
\hline $\begin{array}{l}\text { Respiratory rate } \\
\text { (RR) (normal range } \\
12-20 \text { min) }\end{array}$ & $\begin{array}{l}19(18- \\
20)\end{array}$ & $\begin{array}{l}19(18- \\
21)\end{array}$ & $\begin{array}{l}19(18- \\
20)\end{array}$ & $0.002^{b}$ & $19(18-20)$ & $\begin{array}{l}19(18- \\
21)\end{array}$ & $\begin{array}{l}<0.001 \\
b\end{array}$ \\
\hline $\begin{array}{l}\text { Temperature } \\
\text { (normal range } \\
36.1-37.2 \text { C) }\end{array}$ & $\begin{array}{l}38.0(37.1- \\
38.2)\end{array}$ & $\begin{array}{l}38.0 \\
(37.5- \\
38.3)\end{array}$ & $\begin{array}{l}37.9(37.0- \\
38.2)\end{array}$ & $\begin{array}{l}<0.001 \\
b\end{array}$ & $\begin{array}{l}37.9(37.0- \\
38.2)\end{array}$ & $\begin{array}{l}38.1 \\
(37.9- \\
38.3)\end{array}$ & $\begin{array}{l}<0.001 \\
b\end{array}$ \\
\hline \multicolumn{8}{|l|}{$\begin{array}{l}\text { Blood counts } \\
\text { parameters }\end{array}$} \\
\hline $\begin{array}{l}\text { White blood count } \\
\text { (WBC) } \\
\left(\times 10^{3} / \mathrm{mm}^{3} \text { ) }\right. \\
\text { (normal range:4.0- } \\
11.0)\end{array}$ & $\begin{array}{l}6.6(5.5- \\
7.7)\end{array}$ & $\begin{array}{l}7.3(6.2- \\
8.4)\end{array}$ & $\begin{array}{l}6.4(5.4- \\
7.5)\end{array}$ & $\begin{array}{l}<0.001 \\
b\end{array}$ & $\begin{array}{l}6.4(5.4- \\
7.5)\end{array}$ & $\begin{array}{l}7.6(6.5- \\
8.7)\end{array}$ & $\begin{array}{l}<0.001 \\
b\end{array}$ \\
\hline $\begin{array}{l}\text { Neutrophils } \\
(\mathrm{NEUT})\left(\times 10^{3} /\right. \\
\left.\mathrm{mm}^{3}\right)(\text { normal } \\
\text { range:2.0-7.0 })\end{array}$ & $\begin{array}{l}4.3(3.6- \\
5.2)\end{array}$ & $\begin{array}{l}4.9(4.1- \\
5.9)\end{array}$ & $\begin{array}{l}4.1(3.5- \\
5.0)\end{array}$ & $\begin{array}{l}<0.001 \\
b\end{array}$ & $\begin{array}{l}4.1(3.5- \\
4.9)\end{array}$ & $\begin{array}{l}5.4(4.5- \\
6.2)\end{array}$ & $\begin{array}{l}<0.001 \\
b\end{array}$ \\
\hline
\end{tabular}




\begin{tabular}{|c|c|c|c|c|c|c|c|}
\hline $\begin{array}{l}\text { Lymphocytes } \\
(\mathrm{LYM})\left(\times 10^{3} /\right. \\
\left.\mathrm{mm}^{3}\right)(\text { normal } \\
\text { range:1.0-3.0 ) }\end{array}$ & $\begin{array}{l}1.5(1.2- \\
1.8)\end{array}$ & $\begin{array}{l}1.4(1.2- \\
1.6)\end{array}$ & $\begin{array}{l}1.5(1.2- \\
1.8)\end{array}$ & $0.001^{b}$ & $\begin{array}{l}1.5(1.2- \\
1.8)\end{array}$ & $\begin{array}{l}1.3(1.1- \\
1.5)\end{array}$ & $0.001^{b}$ \\
\hline $\begin{array}{l}\text { Neutrophil-to- } \\
\text { lymphocyte ratio } \\
(\mathrm{NLR})(\text { normal } \\
\text { range: } 0.78-3.53)\end{array}$ & $\begin{array}{l}2.7(2.5- \\
3.5)\end{array}$ & $\begin{array}{l}3.4(2.7- \\
4.6)\end{array}$ & $\begin{array}{l}2.6(2.5- \\
3.2)\end{array}$ & $\begin{array}{l}<0.001 \\
b\end{array}$ & $\begin{array}{l}2.6(2.5- \\
3.1)\end{array}$ & $\begin{array}{l}4.6(3.5 \\
-4.8)\end{array}$ & $\begin{array}{l}<0.001 \\
b\end{array}$ \\
\hline $\begin{array}{l}\text { Platelets }(\mathrm{PLT}) \\
\left(\times 10^{3} / \mathrm{mm}^{3}\right) \\
(\text { normal range: } 150- \\
450)\end{array}$ & $\begin{array}{l}184(141- \\
244)\end{array}$ & $\begin{array}{l}176.0 \\
(136- \\
225)\end{array}$ & $\begin{array}{l}185.0(142 \\
-247)\end{array}$ & $0.024^{b}$ & $\begin{array}{l}185.0(142 \\
-246)\end{array}$ & $\begin{array}{l}175.5 \\
(140- \\
226)\end{array}$ & $0.209^{b}$ \\
\hline $\begin{array}{l}\text { Hemoglobin }(\mathrm{Hb}) \\
\text { (×gr/dL) (normal } \\
\text { range:[Males:13.5- } \\
\text { 17.5], } \\
\text { [Females:12.0- } \\
15.5] \text { ) }\end{array}$ & $\begin{array}{l}13.3(11.9 \\
-14.6)\end{array}$ & $\begin{array}{l}13.0 \\
(11.7- \\
14.4)\end{array}$ & $\begin{array}{l}13.3(11.9- \\
14.6)\end{array}$ & $0.011^{b}$ & $\begin{array}{l}13.3(11.9- \\
14.6)\end{array}$ & $\begin{array}{l}13.2 \\
(11.9- \\
14.5)\end{array}$ & $0.598^{b}$ \\
\hline
\end{tabular}

\section{Inflammatory markers}

\begin{tabular}{|c|c|c|c|c|c|c|c|}
\hline $\begin{array}{l}\text { C-reactive protein } \\
(\mathrm{CRP})(\times \mathrm{mg} / \mathrm{L}) \\
(\text { normal range } \leq 10 \\
)^{2}\end{array}$ & $\begin{array}{l}51.0(26.0 \\
-73.8)\end{array}$ & $\begin{array}{l}56.0 \\
(29.0- \\
85.8)\end{array}$ & $\begin{array}{l}50.0(25.0- \\
72.0)\end{array}$ & $\begin{array}{l}<0.001 \\
b\end{array}$ & $\begin{array}{l}50.7(26.0- \\
72.0)\end{array}$ & $\begin{array}{l}55.5 \\
(26.3- \\
86.0)\end{array}$ & $0.006^{b}$ \\
\hline $\begin{array}{l}\text { Erythrocyte } \\
\text { Sedimentation } \\
\text { Rate (ESR) }\end{array}$ & $\begin{array}{l}39.0(25.0 \\
-63.8)\end{array}$ & $\begin{array}{l}73.0 \\
(37.3- \\
84.0)\end{array}$ & $\begin{array}{l}35.5(25.0 \\
-52.0)\end{array}$ & $\begin{array}{l}<0.001 \\
b\end{array}$ & $\begin{array}{l}37.0(25.0- \\
56.0)\end{array}$ & $\begin{array}{l}68.0 \\
(34.0- \\
84.0)\end{array}$ & $\begin{array}{l}<0.001 \\
b\end{array}$ \\
\hline
\end{tabular}

$(x \mathrm{~mm} / \mathrm{hr})($ normal

range:[Males $\leq 20]$,

[Females: $\leq 30]$ ])

Erythrocyte

Sedimentation

Rate (ESR)

\section{Biochemical}

Parameters

$\begin{array}{llllllll}\text { Serum creatinine } & 1.1(1.0- & 1.2(1.0- & 1.1(1.0- & 0.192^{\mathrm{b}} & 1.1(1.0- & 1.2(1.0- & 0.382^{\mathrm{b}} \\ (\mathrm{CR})(\mathrm{mg} / \mathrm{dL}) & 1.4) & 1.5) & 1.4) & & 1.4) & 1.4) & \end{array}$

(normal range:

0.84 to 1.21 )

\begin{tabular}{|c|c|c|c|c|c|c|c|}
\hline $\begin{array}{l}\text { Creatine } \\
\text { phosphokinase } \\
\text { (CPK)(xU/L) } \\
\text { (normal range: } \\
\text { [Males:39-308], } \\
\text { [Females:26-192]) }\end{array}$ & $\begin{array}{l}218(158- \\
315)\end{array}$ & $\begin{array}{l}264.0 \\
(178 .- \\
319)\end{array}$ & $\begin{array}{l}215.0 \\
(156-315)\end{array}$ & $\begin{array}{l}<0.001 \\
b\end{array}$ & $\begin{array}{l}215.0(156 \\
-315)\end{array}$ & $\begin{array}{l}274.0 \\
(178- \\
342)\end{array}$ & $\begin{array}{l}<0.001 \\
b\end{array}$ \\
\hline $\begin{array}{l}\text { High-sensivity } \\
\text { Troponin T(xng/L) } \\
\text { ([Males } \leq 22], \\
\text { [Females } \leq 14] \text { ) }\end{array}$ & $\begin{array}{l}24.6(16.2 \\
-34.6)\end{array}$ & $\begin{array}{l}37.9 \\
(27.3- \\
49.2)\end{array}$ & $\begin{array}{l}22.5(16.2- \\
31.2)\end{array}$ & $\begin{array}{l}<0.001 \\
b\end{array}$ & $\begin{array}{l}23.1(16.2- \\
33.8)\end{array}$ & $\begin{array}{l}37.4 \\
(27.4- \\
47.1)\end{array}$ & $\begin{array}{l}<0.001 \\
b\end{array}$ \\
\hline
\end{tabular}




\begin{tabular}{|c|c|c|c|c|c|c|c|}
\hline $\begin{array}{l}\text { prothrombin time } \\
\text { (PT)(seconds) } \\
\text { (normal range:11- } \\
13.5 \text { ) }\end{array}$ & $\begin{array}{l}15.0(14.0 \\
-16.2)\end{array}$ & $\begin{array}{l}15.6 \\
(14.4- \\
17.0)\end{array}$ & $\begin{array}{l}14.9(13.9- \\
16.0)\end{array}$ & $\begin{array}{l}<0.001 \\
b\end{array}$ & $\begin{array}{l}14.9(14.0 \text { - } \\
16.1)\end{array}$ & $\begin{array}{l}15.3 \\
(14.3- \\
16.9)\end{array}$ & $\begin{array}{l}<0.001 \\
b\end{array}$ \\
\hline $\begin{array}{l}\text { Partial } \\
\text { Thromboplastin } \\
\text { Time (PTT) } \\
\text { (seconds)(normal } \\
\text { range:25-35) }\end{array}$ & $\begin{array}{l}35.0(30.0- \\
39.4)\end{array}$ & $\begin{array}{l}37.0 \\
(30.6- \\
40.2)\end{array}$ & $\begin{array}{l}34.2(30.0- \\
39.1)\end{array}$ & $\begin{array}{l}<0.001 \\
b\end{array}$ & $\begin{array}{l}34.6(30.0- \\
39.1)\end{array}$ & $\begin{array}{l}37.0 \\
(30.8- \\
40.3)\end{array}$ & $\begin{array}{l}<0.001 \\
b\end{array}$ \\
\hline $\begin{array}{l}\text { international } \\
\text { normalized ratio } \\
\text { (INR) (normal } \\
\text { range:0.8-1.1) }\end{array}$ & $\begin{array}{l}1.3(1.2- \\
1.4)\end{array}$ & $\begin{array}{l}1.3(1.2- \\
1.4)\end{array}$ & $\begin{array}{l}1.3(1.1- \\
1.4)\end{array}$ & $0.021^{b}$ & $\begin{array}{l}1.3(1.2- \\
1.4)\end{array}$ & $\begin{array}{l}1.3(1.2- \\
1.4)\end{array}$ & $0.859^{b}$ \\
\hline \multicolumn{8}{|l|}{ Liver function test } \\
\hline $\begin{array}{l}\text { Alanine } \\
\text { aminotransferase } \\
(\text { ALT)(U/L) (normal } \\
\text { range:10-45) }\end{array}$ & $\begin{array}{l}34.0(25.0 \\
-49.0)\end{array}$ & $\begin{array}{l}52.0 \\
(34.0- \\
62.8)\end{array}$ & $\begin{array}{l}31.0(25.0- \\
42.0)\end{array}$ & $\begin{array}{l}<0.001 \\
\mathrm{~b}\end{array}$ & $\begin{array}{l}31.0(25.0- \\
43.0)\end{array}$ & $\begin{array}{l}51.0 \\
(34.0- \\
61.0)\end{array}$ & $\begin{array}{l}<0.001 \\
b\end{array}$ \\
\hline $\begin{array}{l}\text { Aspartate } \\
\text { aminotransferase } \\
(\mathrm{AST})(\mathrm{U} / \mathrm{L}) \\
\text { (normal range:10- } \\
40 \text { ) }\end{array}$ & $\begin{array}{l}39.0(30.0 \\
-52.0)\end{array}$ & $\begin{array}{l}58.5 \\
(37.0- \\
75.0)\end{array}$ & $\begin{array}{l}37.0(29.0- \\
49.0)\end{array}$ & $\begin{array}{l}<0.001 \\
b\end{array}$ & $\begin{array}{l}37.0(29.0- \\
49.0)\end{array}$ & $\begin{array}{l}59.0 \\
(40.0- \\
76.0)\end{array}$ & $\begin{array}{l}<0.001 \\
b\end{array}$ \\
\hline
\end{tabular}

Note: Data were expressed as mean (standard deviation) and median (IQR) for symmetric and asymmetric numeric variables. Categorical variables were shown as No. (\%). P-values denoted a) the comparison of mean variables between survivor and non-survivor cases using independent $t$-test, $b$ ) the mean rank difference of variables between survivor and non-survivor using Mann Whitney test, and c) the relation between baseline variables and survivor/non-survivor patients using Pearson chi-squared. * Pregnancy percentage was calculated among females.

The most prevalent comorbidity seen in Hypertension (HTN) (31.3\%), Diabetes Mellitus (DM) (27.5\%), Hyperlipidemia (HLP) (13.0\%), and Ischemic heart disease (IHD) (10.5\%). An overlap was seen in Fig. 1.B1 among patients where for example, 14 of them had Hypertension (HTN), Diabetes Mellitus (DM), and Chronic Obstructive Pulmonary Disease (COPD) in their past medical history. The most prevalent comorbidities was recorded among 51-80 years old patients, especially 61-70 years old ones (Fig. 1.B2).

Patients hospitalization was occurred a mean of $7.4 \pm 3.7$ days after the onset of symptoms. The most common symptoms of patients were dyspnea (78.8\%), cough (75.7\%), fever (58.3\%), fatigue (23.7\%), and myalgia or arthralgia (23.5\%). 927(37.5\%) concurrent fever, cough, and dyspnea, and also 13 (0.52\%) presented all intestinalrelated manifestation upon admission day (Fig. 1.A).

At triage, vital signs included oxygen saturation, respiratory rate, and temperature were reported as a median of 92 $\%(88-95), 19(18-20)$, and $38.0^{\circ} \mathrm{C}$ (37.1-38.2), respectively while $92.3 \%$ of them underwent oxygen therapy. Higher Blood pressure, Pulse Rate, Respiratory Rate, and Temperature and lower Oxygen Saturation were detected among death cases. 
Median white blood cell was reported 6600 per micro liter (5500-7700) with the median of 1500 per micro liter (1200-1800) for lymphocytes. Concentrations of C-reactive protein (median: 51.0 (26.0-73.8)), Erythrocyte Sedimentation Rate (median: 39.0 (25.0-63.8)), high-sensitivity Troponin (median: 24.6 (16.2-34.6)) were elevated in most patients. Significantly Higher range of inflammatory markers, coagulation profile, and liver function tests were observed among serious patients.

Spiral chest CT-scan was taken from all participants that reported by the radiologist in which Just 144 (5.8\%) of patients had normal radiological findings. Most of the patients (94.2\%) presented radiological features bilaterally. The most frequent features reported by the radiologists were bi lateral peripheral patchy Ground Glass Opacities (GGO) (94.2\%) and consolidations (29.5\%).

Table 2 described treatment protocols and their association with survival and mortality. During hospitalization, patients were supported by respiratory facilities, 555(22.5\%) of whom by means of nasal cannula, 287(11.6\%) via simple facemask, $1437(58.2 \%)$ through face mask with reserve bag, and $210(8.5 \%)$ with the help of intubation.

Table 2. Intervention results of patients with COVID-19 


\begin{tabular}{|c|c|c|c|c|}
\hline \multirow[t]{2}{*}{ Variables } & \multirow{2}{*}{$\begin{array}{l}\text { Total } \\
(n=2468)\end{array}$} & \multicolumn{2}{|c|}{ Disease Outcome } & \multirow[t]{2}{*}{$P$-value } \\
\hline & & $\begin{array}{l}\text { Deceased } \\
(n=424)\end{array}$ & $\begin{array}{l}\text { Survivor } \\
(n=2044)\end{array}$ & \\
\hline Respiratory facilities, No (\%) & & & & $\begin{array}{l}<0.001 \\
c\end{array}$ \\
\hline None or Unknown & $189(7.7)$ & $51(12)$ & $138(6.8)$ & \\
\hline Nasal & $555(22.5)$ & $52(12.3)$ & $503(24.6)$ & \\
\hline Simple face mask & $287(11.6)$ & $44(10.4)$ & $243(11.9)$ & \\
\hline Reserve bag & $\begin{array}{l}1437 \\
(58.2)\end{array}$ & $277(65.3)$ & $\begin{array}{l}1160 \\
(56.8)\end{array}$ & \\
\hline Intubation & $210(8.5)$ & $136(32.1)$ & $74(3.6)$ & \\
\hline \multicolumn{5}{|l|}{ Drug, N (\%) } \\
\hline Hydroxychloroquine (PO) & $\begin{array}{l}2012 \\
(81.5)\end{array}$ & $342(80.7)$ & $\begin{array}{l}1670 \\
(81.7)\end{array}$ & $0.615^{c}$ \\
\hline Kaletra (Lopinavir/ritonavir) (PO) & $\begin{array}{l}1647 \\
(66.7)\end{array}$ & $257(60.6)$ & $1390(68)$ & $0.003^{c}$ \\
\hline Oseltamivir (PO) & $\begin{array}{l}1835 \\
(74.4)\end{array}$ & $226(53.3)$ & $\begin{array}{l}1609 \\
(78.7)\end{array}$ & $\begin{array}{l}<0.001 \\
c\end{array}$ \\
\hline Paracetamol (Inj) & $\begin{array}{l}2015 \\
(81.6)\end{array}$ & $337(79.5)$ & $\begin{array}{l}1678 \\
(82.1)\end{array}$ & 0.206 \\
\hline Naproxen (PO) & $947(38.4)$ & $145(34.2)$ & $802(39.2)$ & $0.052^{c}$ \\
\hline Ribavirin (PO) & $381(15.4)$ & $128(30.2)$ & $253(12.4)$ & $\begin{array}{l}<0.001 \\
c\end{array}$ \\
\hline Pantoprazole (PO) & $\begin{array}{l}1211 \\
(49.1)\end{array}$ & $215(50.7)$ & $996(48.7)$ & $0.458^{c}$ \\
\hline Ceftriaxone (Inj) & $\begin{array}{l}1687 \\
(68.4)\end{array}$ & $262(61.8)$ & $\begin{array}{l}1425 \\
(69.7)\end{array}$ & $0.001^{c}$ \\
\hline Metoclopramide (Inj) & $105(4.3)$ & $14(3.3)$ & $91(4.5)$ & $0.286^{c}$ \\
\hline Ondansetron (Inj) & $419(17)$ & $70(16.5)$ & $349(17.1)$ & $0.778^{c}$ \\
\hline Diphenhydramine (PO) & $651(26.4)$ & $98(23.1)$ & $553(27.1)$ & $0.094^{c}$ \\
\hline Meropenem (Inj) & 904 (36.6) & $227(53.5)$ & $677(33.1)$ & $\begin{array}{l}<0.001 \\
c\end{array}$ \\
\hline Linezolid (PO) & $230(9.3)$ & $69(16.3)$ & $161(7.9)$ & $\begin{array}{l}<0.001 \\
c\end{array}$ \\
\hline Atrovent (Ipratropium bromide) (Inh) & $451(18.3)$ & $94(22.2)$ & $357(17.5)$ & $0.023^{c}$ \\
\hline Atorvastatin (PO) & $447(18.1)$ & $91(21.5)$ & $356(17.4)$ & $0.049^{c}$ \\
\hline
\end{tabular}




\begin{tabular}{|c|c|c|c|c|}
\hline Vancomycin (Inj) & $\begin{array}{l}1151 \\
(46.6)\end{array}$ & $247(58.3)$ & $904(44.2)$ & $\begin{array}{l}<0.001 \\
c\end{array}$ \\
\hline Dexamethasone (Inj) & $337(13.7)$ & $88(20.8)$ & $249(12.2)$ & $\begin{array}{l}<0.001 \\
c\end{array}$ \\
\hline Salbutamol (Inh) & $590(23.9)$ & $121(28.5)$ & 469 (22.9) & $0.014^{\mathrm{c}}$ \\
\hline (Inh) Combivent (Ipratropium bromide/salbutamol) & $296(12)$ & $77(18.2)$ & $219(10.7)$ & $\begin{array}{l}<0.001 \\
c\end{array}$ \\
\hline Pulmicort (Budesonide) (Inh) & 316 (12.8) & $63(14.9)$ & $253(12.4)$ & $0.164^{\mathrm{c}}$ \\
\hline Levofloxacin (PO) & 297 (12) & $47(11.1)$ & $250(12.2)$ & $0.509^{c}$ \\
\hline Enoxaparin sodium (Inj) & 963 (39) & $141(33.3)$ & $822(40.2)$ & $0.007^{c}$ \\
\hline Heparin (Inj) & $373(15.1)$ & $114(26.9)$ & $259(12.7)$ & $\begin{array}{l}<0.001 \\
c\end{array}$ \\
\hline Aspirin (PO) & $290(11.8)$ & 71 (16.7) & $219(10.7)$ & $\begin{array}{l}<0.001 \\
c\end{array}$ \\
\hline $\mathrm{N}$-acetyl cysteine (Inj) & $309(12.5)$ & $65(15.3)$ & $244(11.9)$ & $0.055^{c}$ \\
\hline Clindamycin (PO) & $32(1.3)$ & $6(1.4)$ & $26(1.3)$ & $0.813^{c}$ \\
\hline Azithromycin (PO) & $\begin{array}{l}1624 \\
(65.8)\end{array}$ & $238(56.1)$ & $\begin{array}{l}1386 \\
(67.8)\end{array}$ & $\begin{array}{l}<0.001 \\
c\end{array}$ \\
\hline Dextromethorphan (PO) & $114(4.6)$ & $22(5.2)$ & $92(4.5)$ & $0.539^{c}$ \\
\hline Remdesivir (Inj) & $123(5)$ & $42(9.9)$ & $81(4)$ & $\begin{array}{l}<0.001 \\
c\end{array}$ \\
\hline Clopidogrel (PO) & $88(3.6)$ & $22(5.2)$ & $66(3.2)$ & $0.048^{c}$ \\
\hline Interferon Beta-1A (Inj) & $175(7.1)$ & $41(9.7)$ & $134(6.6)$ & $0.023^{c}$ \\
\hline Fluconazole (PO) & $70(2.8)$ & $14(3.3)$ & $56(2.7)$ & $0.526^{c}$ \\
\hline Prednisolone (PO) & $140(5.7)$ & $36(8.5)$ & $104(5.1)$ & $0.006^{c}$ \\
\hline Promethazine (Inj) & $82(3.3)$ & $12(2.8)$ & $70(3.4)$ & $0.534^{c}$ \\
\hline Seroflo(Fluticasone/salmeterol) (Inh) & $90(3.6)$ & $18(4.2)$ & $72(3.5)$ & $0.470^{c}$ \\
\hline Ciprofloxacine (PO) & $348(14.1)$ & $70(16.5)$ & $278(13.6)$ & $0.117^{c}$ \\
\hline Vitamin B- Complex (Inj) & $266(10.8)$ & $60(14.2)$ & $206(10.1)$ & $0.014^{c}$ \\
\hline Vitamin C (Inj) & $199(8.1)$ & $54(12.7)$ & $145(7.1)$ & $\begin{array}{l}<0.001 \\
c\end{array}$ \\
\hline Vitamin D (Inj) & $139(5.6)$ & $35(8.3)$ & $104(5.1)$ & $0.010^{c}$ \\
\hline
\end{tabular}




\begin{tabular}{|lllll|}
\hline MgSo4 (Inj) & $237(9.6)$ & $41(9.7)$ & $196(9.6)$ & $0.959^{c}$ \\
\hline Dimenhydrinate (PO) & $260(10.5)$ & $37(8.7)$ & $223(10.9)$ & $0.183^{c}$ \\
\hline Selenium (PO) & $65(2.6)$ & $26(6.1)$ & $39(1.9)$ & $\begin{array}{c}c \\
\text { c }\end{array}$ \\
\hline Clidinium-C (PO) & & & & \\
\hline Bromhexine (PO) & $55(2.2)$ & $4(0.9)$ & $51(2.5)$ & $0.04^{c}$ \\
\hline CaCo3 (PO) & $102(4.1)$ & $21(5)$ & $81(4)$ & $0.351^{c}$ \\
\hline Amantadin (PO) & $82(3.3)$ & $23(5.4)$ & $59(2.9)$ & $0.008^{c}$ \\
\hline Imipenem (Inj) & $129(5.2)$ & $26(6.1)$ & $103(5)$ & $0.357^{c}$ \\
\hline Piperacillin (Inj) & $150(6.1)$ & $23(5.4)$ & $127(6.2)$ & $0.536^{c}$ \\
\hline Atazonavir (PO) & $30(1.2)$ & $6(1.4)$ & $24(1.2)$ & $0.680^{c}$ \\
\hline Dialysis, No. of yes (\%) & $78(3.2)$ & $20(4.7)$ & $58(2.8)$ & $0.044^{c}$ \\
\hline Plasmapheresis, No. of yes (\%) & $75(3)$ & $40(9.4)$ & $35(1.7)$ & $<0.001^{c}$ \\
\hline Plateletet transfusion, No. of yes (\%) & $28(1.1)$ & $17(4)$ & $11(0.5)$ & $\begin{array}{l}<0.001^{c} \\
\text { c }\end{array}$ \\
\hline Blood transfusion, No. of yes (\%) & $8(0.3)$ & $1(0.2)$ & $7(0.3)$ & $0.725 \mathrm{c}$ \\
\hline
\end{tabular}

Note: Data were expressed as mean (standard deviation) and median (IQR) for symmetric and asymmetric numeric variables. Categorical variables were shown as No. (\%). P-values denoted a) the comparison of mean variables between survivor and non-survivor cases using independent $t$-test, $b$ ) the mean rank difference of variables between survivor and non-survivor using Mann Whitney test , and c) the relation between baseline variables and survivor/non-survivor patients using Pearson chi-squared. PO = per os (orally), Inj = injection, Inh = inhalation. Antibacterial agents were prescribed for most of patients, namely, Ceftriaxone for $1687(68.4 \%)$, Meropenem for 904(36.6\%), and Vancomycin for $1151(46.6 \%)$ patients.

Besides, Antiviral agents also were advised for most patients, predominantly, $2012(81.5 \%)$ received Hydroxychloroquine, 1835(74.4\%) received Oseltamivir, 1647(66.7\%) received Kaletra, 381(15.4\%) received Ribavirin, and 123(5\%) received Remdesivir. Glucocorticoids were applied to patients including Dexamethasone in 337 (13.7\%) and Prednisolone in 140(5.7\%). Furthermore, Immunomodulators such as Interferons were administered for $157(7.1 \%)$ of cases. Therapeutic procedures like kidney replacement therapy and Plasmapheresis were performed for $3 \%$ and $1.1 \%$ of critically ill patients, respectively.

The most remarkable adverse events and outcomes of COVID-19 is presented in Table 3 . Weight loss $(9.3 \%)$ defined as $\geq 5 \%$ in comparison to initial weight, myocardial injuries (6.6\%) diagnosed by triple-elevation of Troponin level and electrocardiogram and echocardiogram findings, kidney injury (6\%), and abnormal bleeding (5.1\%) were reported as the most frequent adverse events resulted from COVID-19. During the study, 424 (17.2\%) of patients 
died in hospital following a median of 5 days (3-10) hospitalization. This range included 220 (61.8\%) of 356 patients and 204 (9.7\%) of 2112 patients who were admitted to Intensive Care Unit(ICU) and general ward, respectively. 2044(82.8\%) of patients were discharged alive after a median of 5 days (3-10) of hospitalization. During the 30-day post-discharge follow-up, 148(6.0\%) of 2468 patients readmitted and 25(1.2\%) of 2034 discharged cases died. Full recovery from symptoms after discharge took a median of 14(10-18) days, although the manifestation remained in $5.3 \%$ of cases for more than 30 days. Our results revealed that just 26 (5.8\%) of 450 Cardiopulmonary resuscitations (CPR) were successful. 
Table 3

Event results of patients with COVID-19

Variables

Total

( $n=2468)$

Readmission ( $\leq 30$ days post-discharge), No. of yes (\%)

148 (6.0)

Death in hospital, No. of yes (\%)

$424(17.2)$

Death out of hospital ( $\leq 30$ days post-discharge), No. of yes (\%)

$25 / 2034(1.2) *$

Death in general ward, No. of yes (\%)

204/2112 (9.7)

Death in ICU, No. of yes (\%)

220/356 (61.8)

CVA (ischemic type), No. of yes (\%)

41 (1.7)

Myocardial infarction, No. of yes (\%)

$36(1.5)$

DVT, No. of yes (\%)

$69(2.8)$

Kidney injury, No. of yes (\%)

$148(6)$

Alopecia, No. of yes (\%)

Myocardial injury, No. of yes (\%)

$164(6.6)$

Abnormal bleeding, No. of yes (\%)

$126(5.1)$

Weight loss, No. of yes (\%)

$187(9.3)^{\star \star}$

Skin problems (Acro-ischemic lesions), No. of yes (\%)

$44(1.8)$

Duration of hospitalization, median (IQR)

Total with death

$5.0(3.0-10.0)$

Total with discharge

$5.0(3.0-7.0)$

General with death

$4.0(2.0-6.0)$

General with discharge

$5.0(3.0-7.0)$

ICU with death

$5.0(2.0-9.0)$

ICU with discharge

$5.0(2.0-7.8)$

Successful CPR in hospital, No. of yes (\%)

$26 / 450(5.8)^{\star \star \star ~}$

Full time recovery of symptoms after discharge

Note: Data were expressed as mean (standard deviation) and median (IQR) for symmetric and asymmetric numeric variables. Categorical variables were shown as No. (\%). P-values denoted a) the comparison of mean variables between survivor and non-survivor cases using independent t-test, $b$ ) the mean rank difference of variables between survivor and non-survivor using Mann Whitney test, and c) the relation between baseline variables and survivor/non-survivor patients using Pearson chi-squared. * All deaths out of hospital were occurred between discharge patients; $\star \star$ all weight loss cases are related to patients who survived. *** The

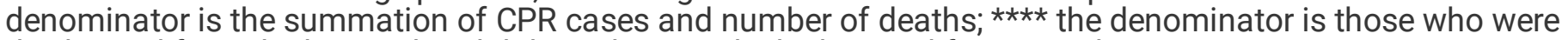
discharged from the hospital and did not die outside the hospital for a month. ICU = intensive care unit, CVA = cerebrovascular accident,

DVT = deep venous thrombosis, $\mathrm{CPR}=$ cardiopulmonary resuscitation 


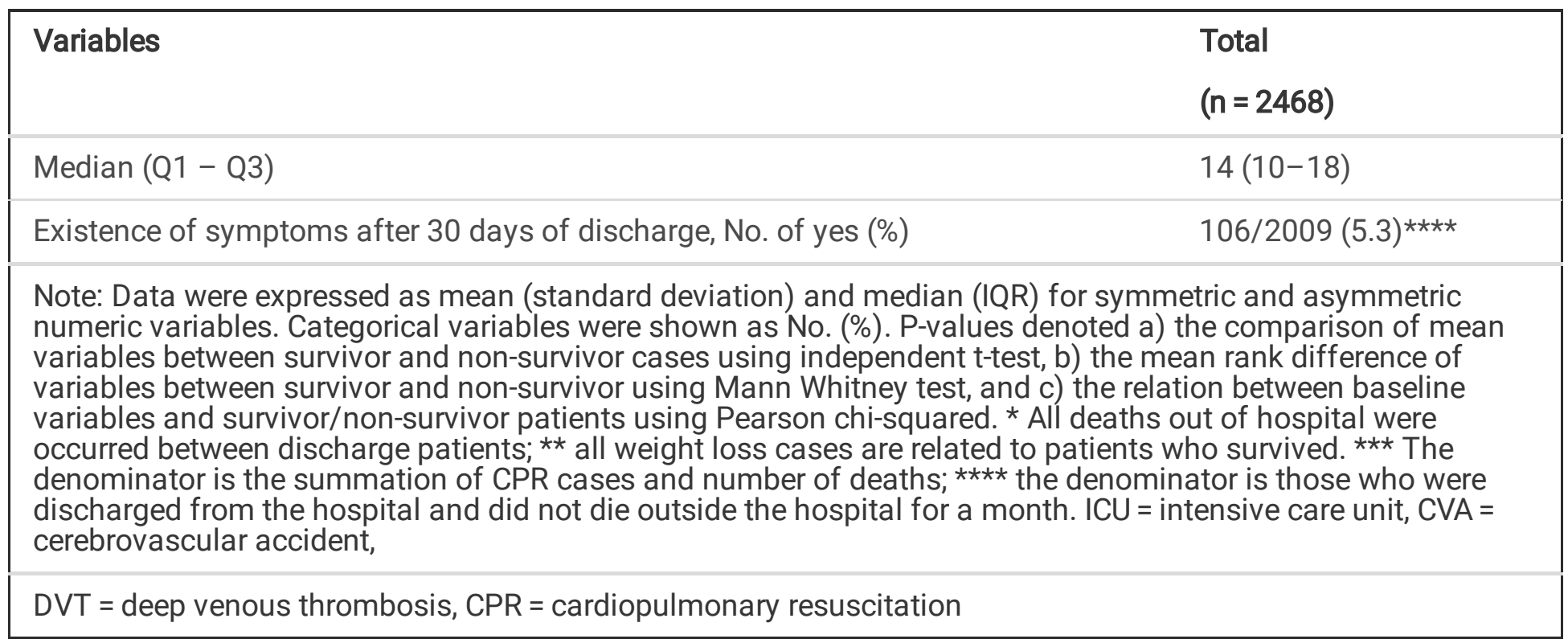

On single and multiple Cox regression model, the significant risk factors of death due to COVID-19 like age, gender, a number of comorbidities, symptoms, Para clinical data and treatment protocols were found, presented in detail in Appendix 1.

The analysis of the final Cox regression model (Fig. 2) revealed that the hazard of death due to COVID-19 significantly increased by more than five-fold per each year of increase in $70-79$ age group $(\mathrm{HR}=5.25,95 \% \mathrm{Cl}: 2.79$, 9.87 ), by $74 \%$ in smokers ( $\mathrm{HR}=1.74,95 \% \mathrm{Cl}: 1.06,2.85)$, and by nearly three-fold in patients with congestive heart failure $(\mathrm{HR}=2.92,95 \% \mathrm{Cl}: 1.44,5.95)$. The therapeutic agents significantly decreased risk of death, by $74 \%$ with Lopinavir/Ritonavir ( $\mathrm{HR}=0.74,95 \% \mathrm{Cl}: 0.60,0.92), 35 \%$ with Oseltamivir ( $\mathrm{HR}=0.35,95 \% \mathrm{Cl}: 0.29,0.43), 66 \%$ with Azithromycin ( $\mathrm{HR}=0.66,95 \% \mathrm{Cl}: 0.53,0.82)$, and more than half among patients who received Interferon $(\mathrm{HR}=0.55$, $95 \% \mathrm{Cl}: 0.39,0.77)$. On the contrary, surprisingly, the hazard of death increased by more than two-fold when it comes to plasmapheresis $(\mathrm{HR}=2.37,95 \% \mathrm{Cl}: 1.39,4.03)$, and by $32 \%$ and $34 \%$ in patients who received Aspirin $(\mathrm{HR}=1.32$, $95 \% \mathrm{Cl}: 1.02,1.71)$ and Ribavirin ( $\mathrm{HR}=1.34,95 \% \mathrm{Cl}: 1.05,1.70)$, respectively. Notably, the hazard ratio of each factor was assessed considering the effect of all other variables.

Figure 3 showed the Kaplan-Meier survival curve with the $95 \%$ confidence interval and representation of the censoring time. Accordingly, the survival probability was 0.14 and 0.12 in the two groups of $<59$ and $\geq 59$ years, respectively, after thirty days of hospitalization.

\section{Discussion}

To the extent of our knowledge, this is the first cohort study of COVID-19 in the case of gathered precise information from admitted patients in the first center of SARS-CoV-19 in Iran. 2468 COVID-19 patients were admitted to four hospitals of Qom during the first months of the Iran's COVID-19 outbreak. Our findings provided informative and stringent demographic data, Para clinical findings, therapeutic approaches, disease outcomes, and post-discharge follow-up in details. However, multivariable analysis were performed to define the risk factors of death subject to COVID-19.

The majority of patients were men having the mean age of $57.9 \pm 17.6$ years and hypertension and diabetes, nearly half of which had upper than normal weight, which was in line with China [8] and USA pattern [5]. The most prevalent symptoms were dyspnea (78.8\%), cough (75.7\%), and fever (58.3\%), which are consistent with Ashraf et 
al [2] and Guan et al[9]. These data may represent fever as a minor symptom of COVID-19, whilst cough and dyspnea are major ones. $92.3 \%$ of patients received respiratory facilities while $14.42 \%$ of whom were admitted to ICU. In the course of the study, $17.2 \%$ and $1.2 \%$ of patients died in hospital and out of hospital, respectively, which is similar to Germany and France[7], but lower than UK with 39\% of mortality[10]. Surely, the inconsistency result ascribes insignificant differences between countries in population demographic data and health care systems. The mortality rate was reported $2.3 \%$ at the onset of COVID-19 epidemic [11]. This controversy may result from investigating the patients until 30-days after discharge, and just admitted patients were participated.

$356(14.42 \%)$ out of 2468 participants were critically ill and admitted to ICU, which is similar to reports from china $[9,12]$, Italy [13], and New York City [14]. 92.3\% of patients required respiratory facilities which is consistent with Italy reports, but it is higher than China[8, 9, 12, 15], New York[14], and Washington state [16]. These high rates of critically ill patients requiring respiratory support and ICU admission, emphasizes the severity of the disease in Iran and urgent need for Iran's hospital conveniences. .

In this cohort study, 424(17.2\%) patients died in hospital and 2044(82.8\%) were discharged. The significant risk factors of death related to COVID-19 were ageing, male gender, HTN, CHF, CVA, CKD, increasing ESR, PT, WBC, liver function tests, and decreasing Oxygen saturation. Particularly, our data showed the higher survival rate for younger than 59 years, which is in line with Germany reports [7]. Similarly, Cummings et al reported that older age, underlying cardiopulmonary diseases, and higher ranges of D-dimer, ALT, CRP, and Troponin are regarded as risk factors of poor outcomes [14]. The abnormal level of Prothrombin might result from procoagulant state in COVID19 [17]. Abnormal serum Creatinine levels might be secondary to direct kidney injury or fluid imbalance, and higher levels of WBC might be a clue of bacterial super-infection.

Similarly to data from China [12] and Italy [18], Hypertension was associated with poor in-hospital outcomes. In consistent with CVA as a risk factor of death in our study, an analysis of Aggawal G et al revealed a 2.5-fold increase in severity of COVID-19 illness among patients with underlying cerebrovascular disease [19].

$55.2 \%$ of admitted patients were overweight while critically deceased cases had higher BMI mean in comparison with the survivors. These data are similar to UK[20] and USA[14], where obesity has been related with higher rates of ICU admission and mortality. Although obesity is an exacerbating factor for many diseases including HTN, DM, CVA, liver and renal dysfunctions, confirming studies needs to be performed to approve that association.

Non-survivors had higher range of Blood pressure, Temperature, Pulse rate, Respiratory rate, and lower Oxygen saturation compared to survivors. These findings illustrates that patients' abnormal vital signs might be prognostic factors of severity.

Anti-viral agents were administered to all patients. Lopinavir/Ritonavir and Remdesivir played a significant role in patients' survival. Ashraf et al reported the positive effect of Kaletra on patients' outcomes[2]. It should be noted that the US National Institute of Allergy and Infectious Diseases approved an emergency administration of Remdesivir for critical cases of COVID-19 inpatients. The efficacy of administrating Hydroxychloroquine with respect to COVID-19 patients remains to be understood. The revealed information from the USA have not disclosed the beneficial effect of Hydroxychloroquine for COVID-19 inpatients[21]. Interferon was one of the Immunomodulators with remarkable effects on COVID-19 patients' survival in our study. Interferons (IFN) strengthen the immune system by antiviral and immunomodulatory activities[22]. Nile et al reported the benefits of IFNs against SARS-CoV-2, alone or in combination with the other anti-viral agents[23].The most challenging therapeutic agent would be plasmapheresis, which increases the hazard of death on the contrary with other studies 
in which it has reported the beneficial effect of a COVID-19 convalescent plasma transfusion on the treatment of critically ill COVID-19 cases through neutralization of SARS-CoV-2 and inhibition of cytokine storm[24-26]. However, we evaluated this effect beside other co-factors, and just $1.1 \%$ of the participants underwent plasmapheresis, therefore, it requires further studies with larger study population.

One of the most striking features of this study would be a 30 -day post-discharge follow-up indicated $6.0 \%$ readmission, $1.2 \%$ post-discharge death, the median of 14 days of recovery of symptoms, and existence of symptoms in $5.3 \%$ of patients after 30 days of discharge. Similarly, Ashraf et al reported $8.6 \%$ of readmission and $4.3 \%$ of death after discharge[2]. These data emphasizes the symptoms relapse and the importance of close followup after discharge.

To our knowledge, this study is the first study of COVID-19 with detailed information of participants in Qom, The first city in Middle East in which COVID-19 was diagnosed.

To wrap up, various outcomes of COVID-19 among different countries attribute to different demographic data and health care systems. This disease has the potential to aggravate with regard to identified risk factors. As a result, atrisk patients need to be looked after specifically in favor of saving more lives.

\section{Declarations}

\section{Data sharing statement}

Data would be available upon reasonable request by contacting the corresponding author.

\section{Authors' contributions}

MAP, HY and SHA developed the original idea for the study. HY, HF, NN, ZH, MY, ZS, MA and EM contributed in data gathering, data extraction and checking the validity of laboratory results in documents. MAP and MAL conducted the analysis. NT, MAP HY wrote the first draft of the manuscript. MA and SHA revised manuscript. All authors reviewed and accepted the paper prior to submission.

\section{Declaration of Competing Interest}

None to declare.

\section{Acknowledgments}

The authors would like to thank Qom University of Medical sciences for access to the patient's documents and medical records.

\section{Funding:}

This work was supported by Qom University of Medical sciences

Conflict of Interest: No conflict of interest for all authors

\section{References}


1. Zhu N, Zhang D, Wang W, et al. A Novel Coronavirus from Patients with Pneumonia in China, 2019. N Engl J Med 2020;382:727-33.

2. Ashraf MA, Shokouhi N, Shirali E, et al. COVID-19 in Iran, a comprehensive investigation from exposure to treatment outcomes. medRxiv 2020.

3. Covid W. coronavirus pandemic. 2020.

4. Arab-Mazar Z, Sah R, Rabaan AA, et al. Mapping the incidence of the COVID-19 hotspot in Iran-Implications for Travellers. Travel Medicine and Infectious Disease 2020.

5. Richardson S, Hirsch JS, Narasimhan M, et al. Presenting characteristics, comorbidities, and outcomes among 5700 patients hospitalized with COVID-19 in the New York City area. Jama 2020.

6. Khoshnood RJ, Ommi D, Zali A, et al. Epidemiological Characteristics, Clinical Features, and Outcome of COVID-19 Patients in Northern Tehran, Iran; a Cross-Sectional Study. Advanced Journal of Emergency Medicine 2020.

7. Karagiannidis C, Mostert C, Hentschker C, et al. Case characteristics, resource use, and outcomes of 10021 patients with COVID-19 admitted to 920 German hospitals: an observational study. The Lancet Respiratory Medicine 2020;8:853-62.

8. Yang $X, Y u Y, X u$ J, et al. Clinical course and outcomes of critically ill patients with SARS-CoV-2 pneumonia in Wuhan, China: a single-centered, retrospective, observational study. Lancet Respir Med 2020;8:475-81.

9. Guan WJ, Ni ZY, Hu Y, et al. Clinical Characteristics of Coronavirus Disease 2019 in China. N Engl J Med 2020;382:1708-20.

10. Docherty AB, Harrison EM, Green CA, et al. Features of 20133 UK patients in hospital with covid-19 using the ISARIC WHO Clinical Characterisation Protocol: prospective observational cohort study. bmj 2020;369.

11. Novel CPERE. The epidemiological characteristics of an outbreak of 2019 novel coronavirus diseases (COVID19) in China. Zhonghua liu xing bing xue za zhi= Zhonghua liuxingbingxue zazhi 2020;41:145.

12. Wu Z, McGoogan JM. Characteristics of and important lessons from the coronavirus disease 2019 (COVID-19) outbreak in China: summary of a report of 72314 cases from the Chinese Center for Disease Control and Prevention. Jama 2020;323:1239-42.

13. Giacomo G, Antonio P, Cecconi M. Critical care utilization for the COVID-19 outbreak in Lombardy, Italy. 2020.

14. Cummings MJ, Baldwin MR, Abrams D, et al. Epidemiology, clinical course, and outcomes of critically ill adults with COVID-19 in New York City: a prospective cohort study. The Lancet 2020.

15. Zhou F, Yu T, Du R, et al. Clinical course and risk factors for mortality of adult inpatients with COVID-19 in Wuhan, China: a retrospective cohort study. Lancet 2020;395:1054-62.

16. Arentz M, Yim E, Klaff L. Characteristics and outcomes of 21 critically ill patients with COVID-19 in Washington State [published online March 19, 2020]. JAMA doi;10.

17. Ranucci M, Ballotta A, Di Dedda U. The procoagulant pattern of patients with COVID-19 acute respiratory distress syndrome.[published online ahead of print, 2020 Apr 17]. J Thromb Haemost.

18. Grasselli G, Zangrillo A, Zanella A, et al. Baseline Characteristics and Outcomes of 1591 Patients Infected With SARS-CoV-2 Admitted to ICUs of the Lombardy Region, Italy. Jama [Internet]. 2020; 1-8. 2020.

19. Aggarwal G, Lippi G, Michael Henry B. Cerebrovascular disease is associated with an increased disease severity in patients with Coronavirus Disease 2019 (COVID-19): A pooled analysis of published literature. International Journal of Stroke 2020;15:385-9.

20. Audit I. ICNARC report on COVID-19 in critical care. April 2020;10:2020.

Page $21 / 25$ 
21. Geleris J, Sun Y, Platt J, et al. Observational Study of Hydroxychloroquine in Hospitalized Patients with Covid19. N Engl J Med 2020;382:2411-8.

22. Wang BX, Fish EN, editors. Global virus outbreaks: Interferons as 1st responders. Seminars in immunology; 2019: Elsevier.

23. Nile SH, Nile A, Qiu J, et al. COVID-19: Pathogenesis, cytokine storm and therapeutic potential of interferons. Cytokine Growth Factor Rev 2020;53:66-70.

24. Ye M, Fu D, Ren Y, et al. Treatment with convalescent plasma for COVID-19 patients in Wuhan, China. J Med Virol 2020;92:1890-901.

25. Duan K, Liu B, Li C, et al. Effectiveness of convalescent plasma therapy in severe COVID-19 patients. Proceedings of the National Academy of Sciences 2020;117:9490-6.

26. Keith P, Day M, Choe C, et al. The successful use of therapeutic plasma exchange for severe COVID-19 acute respiratory distress syndrome with multiple organ failure. SAGE open medical case reports 2020;8:2050313X20933473.

\section{Figures}



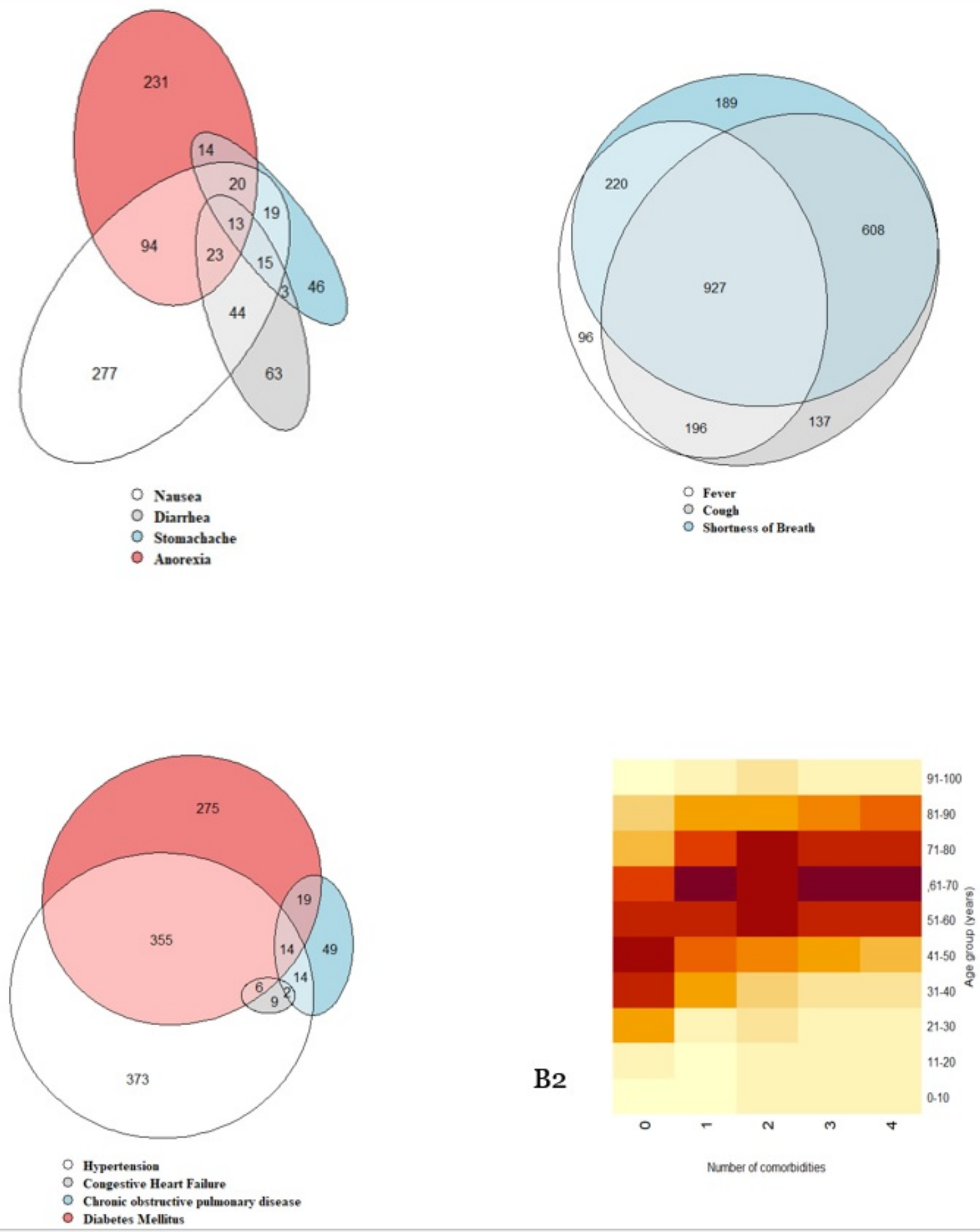

Figure 1

(A). Euler diagram of overlapped symptoms. (B):1. Euler diagram of overlapped comorbidities, 2. Heatmap of number of comorbidities among different age groups. 


\begin{tabular}{|c|c|c|c|c|c|c|}
\hline Variable & & $\mathrm{N}$ & Events & Hazard ratio & & $\mathrm{r}$ \\
\hline \multirow[t]{2}{*}{ Antibiotic } & No & 2335 & 390 & 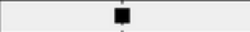 & Reference & \\
\hline & Yes & 133 & 34 & $\square$ & $0.69(0.47,1.00)$ & 0.04 \\
\hline \multirow[t]{2}{*}{ Kaletra } & No & 821 & 167 & & Reference & \\
\hline & Yes & 1647 & 257 & - & $0.74(0.60,0.92)$ & 0.006 \\
\hline \multirow[t]{2}{*}{ Oseltamivir } & No & 633 & 198 & & Reference & \\
\hline & Yes & 1835 & 226 & - & $0.35(0.29,0.43)$ & $<0.001$ \\
\hline \multirow[t]{2}{*}{ Ribavirin } & No & 2087 & 296 & & Reference & \\
\hline & Yes & 381 & 128 & & $1.34(1.05,1.70)$ & 0.018 \\
\hline \multirow[t]{2}{*}{ Ceftriaxone } & No & 781 & 162 & & Reference & \\
\hline & Yes & 1687 & 262 & & $1.04(0.83,1.31)$ & 0.715 \\
\hline \multirow[t]{2}{*}{ Diphenhydramine } & No & 2049 & 354 & & Reference & \\
\hline & Yes & 419 & 70 & & $0.90(0.69,1.17)$ & 0.418 \\
\hline \multirow[t]{2}{*}{ Combivent } & No & 2172 & 347 & & Reference & \\
\hline & Yes & 296 & 77 & -1 & $1.48(1.14,1.92)$ & 0.004 \\
\hline \multirow[t]{2}{*}{ Clexan } & No & 1505 & 283 & 中 & Reference & \\
\hline & Yes & 963 & 141 & -5 & $0.90(0.72,1.14)$ & $0.38 c$ \\
\hline \multirow[t]{2}{*}{ Heparin } & No & 2095 & 310 & & Reference & \\
\hline & Yes & 373 & 114 & & $1.20(0.94,1.52)$ & $0.14 c$ \\
\hline \multirow[t]{2}{*}{ ASA } & No & 2178 & 353 & & Reference & \\
\hline & Yes & 290 & 71 & - & $1.32(1.02,1.71)$ & 0.036 \\
\hline \multirow[t]{2}{*}{ NAC } & No & 2159 & 359 & 中 & Reference & \\
\hline & Yes & 309 & 65 & - & $0.89(0.68,1.18)$ & 0.432 \\
\hline \multirow[t]{2}{*}{ Azithromycin } & No & 844 & 186 & i & Reference & \\
\hline & Yes & 1624 & 238 & - & $0.66(0.53,0.82)$ & $<0.001$ \\
\hline \multirow[t]{2}{*}{ Remdesivir } & No & 2345 & 382 & 中 & Reference & \\
\hline & Yes & 123 & 42 & 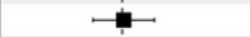 & $1.02(0.72,1.44)$ & 0.923 \\
\hline \multirow[t]{2}{*}{ Interferon } & No & 2293 & 383 & 中 & Reference & \\
\hline & Yes & 175 & 41 & 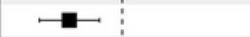 & $0.55(0.39,0.77)$ & $<0.001$ \\
\hline \multirow[t]{2}{*}{ VitC } & No & 2269 & 370 & 亗 & Reference & \\
\hline & Yes & 199 & 54 & 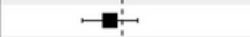 & $0.87(0.63,1.19)$ & 0.38 \\
\hline \multirow[t]{2}{*}{ Selenium } & No & 2403 & 398 & | & Reference & \\
\hline & Yes & 65 & 26 & $\square$ & $1.12(0.71,1.76)$ & 0.632 \\
\hline \multirow[t]{2}{*}{ Plasmapheresis } & No & 2440 & 407 & | & Reference & \\
\hline & Yes & 28 & 17 & $\square$ & $2.37(1.39,4.03)$ & 0.002 \\
\hline
\end{tabular}

\begin{tabular}{|c|c|c|c|c|c|c|}
\hline Variable & & $\mathrm{N}$ & Events & Hazard ratio & & $\mathbf{p}$ \\
\hline \multirow[t]{2}{*}{ Gender } & Female & 936 & 137 & i & Reference & \\
\hline & Male & 1214 & 202 & & $1.16(0.92,1.45)$ & 0.206 \\
\hline \multirow[t]{6}{*}{ Age } & $<39$ & 420 & 17 & 口 & Reference & \\
\hline & $40-49$ & 367 & 46 & $\longmapsto$ & $2.20(1.26,3.84)$ & 0.006 \\
\hline & $50-59$ & 472 & 55 & $\square$ & $1.76(1.01,3.07)$ & 0.044 \\
\hline & $60-69$ & 516 & 104 & $\longmapsto$ & $3.08(1.82,5.20)$ & $<0.001$ \\
\hline & $70-79$ & 67 & 24 & $\longmapsto$ & $5.25(2.79,9.87)$ & $<0.001$ \\
\hline & $>=80$ & 308 & 93 & $\longmapsto$ & $3.39(1.98,5.79)$ & $<0.001$ \\
\hline \multirow[t]{2}{*}{ Smoke } & No & 2045 & 320 & a & Reference & \\
\hline & Yes & 105 & 19 & $\square$ & $1.74(1.06,2.85)$ & 0.027 \\
\hline \multirow[t]{2}{*}{ HTN } & No & 1516 & 207 & & Reference & \\
\hline & Yes & 634 & 132 & & $1.18(0.93,1.50)$ & 0.170 \\
\hline \multirow[t]{2}{*}{$\mathrm{CHF}$} & No & 2128 & 331 & & Reference & \\
\hline & Yes & 22 & 8 & $\longmapsto$ & $2.92(1.44,5.95)$ & 0.003 \\
\hline \multirow[t]{2}{*}{ COPD } & No & 2074 & 320 & & Reference & \\
\hline & Yes & 76 & 19 & $\longmapsto$ & $0.94(0.57,1.56)$ & 0.821 \\
\hline \multirow[t]{2}{*}{ DM } & No & 1581 & 226 & 4 & Reference & \\
\hline & Yes & 569 & 113 & $\mapsto$ & $1.01(0.78,1.29)$ & 0.967 \\
\hline \multirow[t]{2}{*}{ CVA } & No & 2102 & 325 & 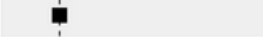 & Reference & \\
\hline & Yes & 48 & 14 & $\longrightarrow$ & $1.58(0.90,2.76)$ & 0.112 \\
\hline \multirow[t]{2}{*}{ HLP } & No & 1891 & 290 & 亗 & Reference & \\
\hline & Yes & 259 & 49 & $\mapsto$ & $1.13(0.83,1.54)$ & 0.429 \\
\hline \multirow[t]{2}{*}{ Tuberculosis } & No & 2102 & 325 & 中 & Reference & \\
\hline & Yes & 48 & 14 & & & \\
\hline
\end{tabular}

Figure 2

Hazard of death due to SARS-CoV-2 


\section{Survival curves}

Based on Kaplan-Meier estimates

Strata $\div<59 \mp>=59$

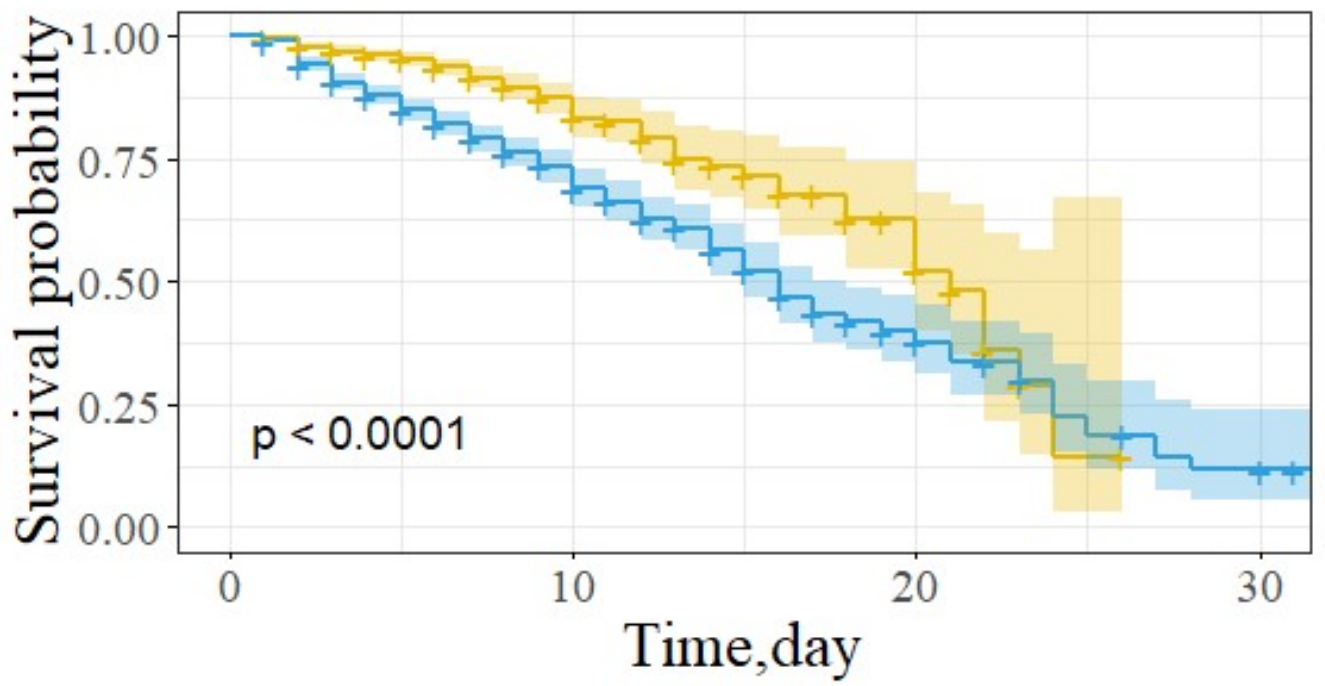

created with survminer

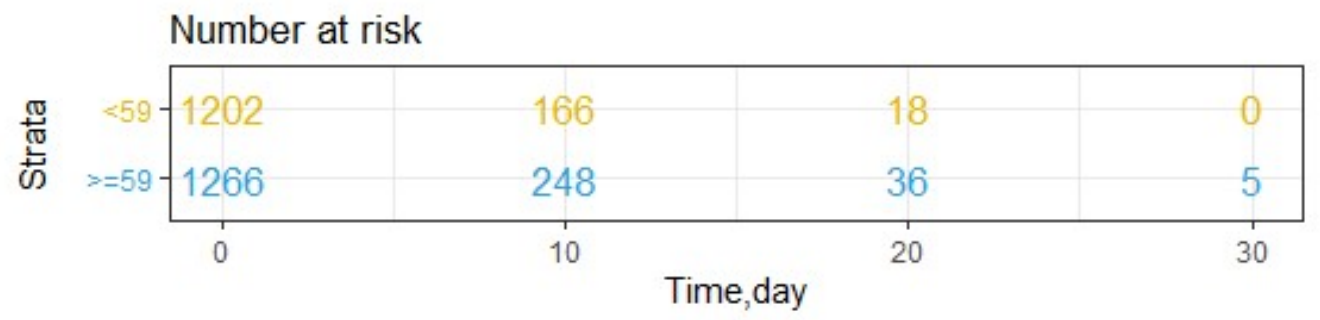

Figure 3

Kaplan-Meier based survival curve according to hospital admission due to COVID-19 among two age groups.

\section{Supplementary Files}

This is a list of supplementary files associated with this preprint. Click to download.

- app.docx 